\title{
On the non-linear energy transfer in a gravity-wave spectrum

\author{
Part 1. General theory
}

\author{
By K. HASSELMANN \\ Institute for Naval Architecture, University of Hamburg $\dagger$
}

(Received 18 August 1961)

The energy flux in a finite-depth gravity-wave spectrum resulting from weak non-linear couplings between the spectral components is evaluated by means of a perturbation method. The fifth-order analysis yields a fourth-order effect comparable in magnitude to the generating and dissipating processes in windgenerated seas. The energy flux favours equidistribution of energy and vanishes in the limiting case of a white, isotropic spectrum. The influence on the equilibrium structure of fully developed wave spectra and on other phenomena in random seas is discussed briefly.

\section{Introduction}

The last years have seen considerable progress in our understanding of the linear processes responsible for the initial growth of wind-generated ocean waves. Comparatively little is still known, however, of the non-linear processes which play an important role in the later stages of development and largely determine the equilibrium state finally attained by the sea. The principal non-linear effects can probably be reduced to two independent processes:

(1) the breaking of waves due to the occurrence of local instability when the downward acceleration of water particles exceeds the gravitational acceleration $g$, and

(2) the energy transfer in the spectrum resulting from non-linear interactions between different wave components.

Although the first process represents one of the principal causes of energy dissipation in the spectrum and is thus of considerable importance, we shall be concerned in this paper only with the second process.

A non-linear transfer of energy between different wave components is to be expected from the general behaviour of coupled mechanical systems. In the case of a wave spectrum, the non-linear couplings are small and can hence be analysed with the aid of conventional perturbation expansions about the known linear solution. The existence of unsteady third-order perturbations representing a continuous transfer of energy between discrete wave components has been demonstrated by Phillips (1960), and the rate of growth of the tertiary wave has since been evaluated explicitly by Longuet-Higgins (1962) for two

$\uparrow$ Present address: Institute of Geophysics and Planetary Physics, University of California, La Jolla. 
intersecting wave trains. In the case of a continuous spectrum, however, we shall find that the perturbation analysis has to be extended further to the fifth order in order to determine the energy flux resulting from the non-steady interactions. The step from the discrete interactions obtained by perturbation analysis of the equations of motion to the energy transfer in a continuous spectrum is then based on certain asymptotic integral formulae ( $\$ 3$ ) which represent, in a sense, the key point of the analysis.

It is known from the turbulence problem that the energy transfer due to non-linear interactions between random components in an energy spectrum cannot be evaluated without further knowledge of the underlying statistical process. In contrast to the turbulence problem, this apparently presents little difficulty for gravity waves, as the interactions are weak, and it is generally assumed (and can, in fact, be deduced from a loose application of the Central Limit Theorem) that in the linear approximation a wind-generated random sea is Gaussian.

The final expression for the energy flux can be interpreted in terms of quadruple interactions in which energy is transferred from three 'active' wave components to a 'passive' fourth component, which receives energy from the 'active' components but has no direct influence on the interaction. The net result of all interactions is to redistribute the energy of the spectrum more uniformly over all wave-numbers, the energy flux vanishing in the limiting case of an isotropic, white spectrum. For wave spectra in the latter stages of development with pronounced maxima at low wave-numbers, this generally leads to an energy flux from the peak to shorter waves, similar to the non-linear energy flux in a turbulence spectrum. Some energy, however, will also flow from the peak to still longer waves, and it has been suggested that this (or perhaps the steady second-order interactions) may be the cause for the extremely long, lowenergy waves observed after severe storms (Munk 1961). As the wavelengths of these waves are comparable with, or greater than, the ocean depth, the analysis has been carried through in the following generally for waves of finite depth.

The characteristic time scale of the energy flux is proportional to the fourth power of the root-mean-square wave slope. $\dagger$ This gives an estimated order of magnitude of the energy flux comparable for fully developed spectra to the generating and dissipating processes in a sea. A rough computation for a typical fully developed spectrum yielded, as expected, an energy transfer from low to high wave-numbers, with characteristic times of a few fractions of an hour for the shorter waves and a few hours for the longer waves (Hasselmann 1961).

\section{Perturbation analysis}

We consider the irrotational motion of a horizontally unbounded ideal fluid of finite constant depth $h$ with a free surface $z=\zeta(x, y, t)$, where $x, y$ and $z$ denote Cartesian co-ordinates, with the $z$-axis directed vertically upwards. Neglecting

$\dagger$ It should be pointed out that this cannot be derived from an analysis of the discrete interactions alone, as these lead to a linear increase in the amplitudes of the perturbations, whereas in a continuous spectrum the energy changes linearly. 
surface tension, the velocity potential $\phi(\mathbf{x}, t)$ and surface deviation $\zeta$ are then determined by the (non-linear) system of equations

$$
\begin{array}{rll}
\nabla^{2} \phi=0 \text { for } z<\zeta & \begin{array}{c}
\text { (continuity equation), } \\
\text { (kinematical boundary condition } \\
\text { at the bottom), }
\end{array} \\
\frac{\partial \zeta}{\partial t}-\frac{\partial \phi}{\partial z}+\tilde{\nabla} \zeta \cdot \tilde{\nabla} \phi=0 \text { for } z=-h \quad z=\zeta & \begin{array}{c}
\text { finematical boundary condition } \\
\text { at the free surface), } \\
\frac{\partial \phi}{\partial t}+g \zeta+\frac{1}{2}(\nabla \phi)^{2}=0 \text { for } z=\zeta
\end{array} \begin{array}{c}
\text { (dynamical boundary condition } \\
\text { at the free surface) }
\end{array}
\end{array}
$$

and

$$
\left.\begin{array}{rl}
\zeta(\tilde{\mathbf{x}}, 0) & =\zeta_{0}(\tilde{\mathbf{x}}) \text { for } t=0, \\
\frac{\partial \phi}{\partial \eta}\left(x, y, \zeta_{0}, 0\right) & =\frac{\partial \phi_{0}}{\partial \eta}(\tilde{\mathbf{x}}) \text { for } z=\zeta_{0}, t=0,
\end{array}\right\} \quad \text { (initial conditions), }
$$

where $\eta$ is the normal at the free surface and $\zeta_{0}$ and $\partial \phi_{0} / \partial \eta$ are the given initial values of the free surface and the normal free-surface velocity, respectively. The tilda $\sim$ denotes two-dimensional vectors or operators in the $x y$-plane.

Assuming small wave slopes, an approximate solution of (1.1)-(1.6) can be obtained by expanding $\phi$ and $\zeta$ in the perturbation series

$$
\begin{aligned}
& \phi={ }_{1} \phi+{ }_{2} \phi+{ }_{3} \phi+\ldots, \\
& \zeta={ }_{1} \zeta+{ }_{2} \zeta+{ }_{3} \zeta+\ldots,
\end{aligned}
$$

where the perturbation parameter (the wave slope) has been drawn into the perturbation functions, the subscripts thus denoting the order of magnitude of the functions. The linear equations (1.1) and (1.2) then yield

$$
\begin{aligned}
& \nabla_{n}^{2} \phi=0 \quad \text { for } \quad z<\zeta, \\
& \frac{\partial_{n} \phi}{\partial z}=0 \quad \text { for } \quad z=-h .
\end{aligned}
$$

Expanding (1.3) and (1.4) in $z$ about the undisturbed free surface $z=0$ and substituting (1.7) and (1.8), we obtain boundary conditions for the perturbation functions at $z=0$,

$$
\begin{gathered}
\frac{\partial_{n} \zeta}{\partial t}-\frac{\partial_{n} \phi}{\partial z}=\sum_{\nu}\left\{\frac{\nu_{1} \zeta_{\nu_{2}} \zeta \cdots \nu_{p-1} \zeta}{(p-1) !} \frac{\partial^{p}}{\partial z^{p}} v_{p} \phi-\frac{{ }_{\nu_{1}} \zeta_{\nu_{2}} \zeta{ }_{\nu_{p-2}} \zeta}{(p-2) !} \tilde{\nabla}_{\nu_{p-1}} \zeta \frac{\partial^{p-2}}{\partial z^{p-2}} \tilde{\nabla}_{\nu_{p}} \phi\right\},(1.11) \\
\frac{\partial_{n} \phi}{\partial t}+g_{n} \zeta=-\sum_{\nu}\left\{\frac{\nu_{1} \zeta_{\nu_{2}} \zeta \cdots \nu_{p-1} \zeta}{(p-1) !} \frac{\partial^{p}}{\partial z^{p-1} \partial t}\left({ }_{\nu_{p}} \phi\right)+\frac{{ }_{\nu_{1}} \zeta_{\nu_{2}} \zeta \cdots \nu_{p-2} \zeta}{2(p-2) !} \frac{\partial^{p-2}}{\partial z^{p-2}}\left[\nabla_{\nu_{p-1}} \phi . \nabla_{\nu_{p}} \phi\right]\right\},
\end{gathered}
$$

where the sums are taken over all combinations of index groups

$$
\nu=\left(\nu_{1}, v_{2}, \ldots, \nu_{p}\right) \text { with } 2 \leqslant p \leqslant n \text { and } \sum_{j=1}^{p} \nu_{j}=n
$$

For $p=2$ the factors $[(p-2) !]^{-1}{ }_{\nu_{1}} \zeta_{\nu_{2}} \zeta \ldots{ }_{\nu_{-2}} \zeta$ in the second terms of the sums are to be taken as unity. 
Instead of (1.11), it is more convenient to use the linear combination $\partial / \partial t(1.12)-g(1.11)$, in which ${ }_{n} \zeta$ has been eliminated:

$$
\begin{aligned}
& \frac{\partial^{2}{ }_{n} \phi}{\partial t^{2}}+g \frac{\partial_{n} \phi}{\partial z}=-\sum_{\nu}\left\{\frac{\partial}{\partial t}\left[\frac{{ }_{\nu_{1}} \zeta_{\nu_{3}} \zeta \cdots \nu_{p-1} \zeta}{(p-1) !} \frac{\partial^{p}}{\partial z^{p-1} \partial t}{ }_{\nu p} \phi+\frac{{ }_{\nu_{1}} \zeta_{\nu_{2}} \zeta \cdots \nu_{p-z} \zeta}{2(p-2) !} \frac{\partial^{p-2}}{\partial z^{p-2}}\left(\nabla_{\nu_{p-1}} \phi \cdot \nabla_{\nu_{p}} \phi\right)\right]\right.
\end{aligned}
$$

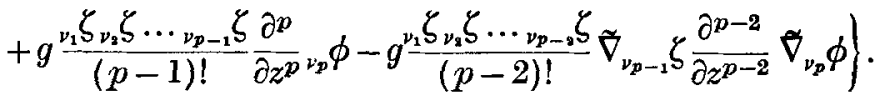

In a similar manner, (1.5) and (1.6), together with (1.4), yield initial conditions

and for $n \geqslant 2$,

$$
\begin{gathered}
\frac{\partial_{1} \phi}{\partial z}=\frac{\partial \phi_{0}}{\partial \eta}(\tilde{\mathbf{x}}) \text { for } z=0, t=0, \\
\frac{\partial_{1} \phi}{\partial t}=-g \zeta_{0}(\tilde{\mathbf{x}}) \text { for } z=0, t=0
\end{gathered}
$$

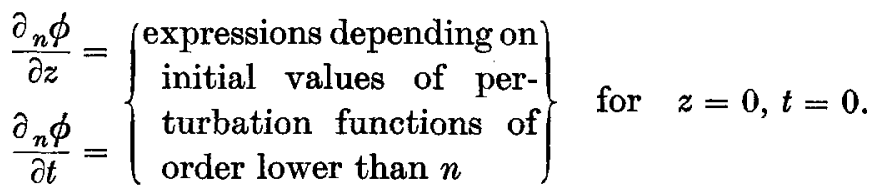

Equations (1.9)-(1.17) are linear in the highest perturbation order and can hence be solved for successive orders.

We consider now the particular initial-value problem in which the initial functions $\partial \phi_{0} / \partial \eta$ and $\zeta_{0}$ are random, homogeneous functions of $\tilde{\mathbf{x}}$. The perturbation solutions ${ }_{n} \phi$ and ${ }_{n} \zeta$ will then also be random and homogeneous in $\tilde{\mathbf{x}}$. They can thus be represented as Fourier-Stieltjes integrals with respect to a two-dimensional wave-number k. Applying (1.9) and (1.10), we then have

$$
\begin{aligned}
{ }_{n} \phi & =\sum_{\mathbf{k}} \phi_{\mathbf{k}}(t) \frac{\cosh k(z+h)}{\cosh k h} \exp [i(\mathbf{k} . \tilde{\mathbf{x}})], \\
{ }_{n} \zeta & =\sum_{\mathbf{k}} Z_{\mathbf{k}}(t) \exp [i(\mathbf{k} \cdot \tilde{\mathbf{x}})]
\end{aligned}
$$

where approximating Fourier sums have been introduced (and will be used throughout) in place of exact Fourier-Stieltjes integrals, as they allow a more condensed presentation of the multiple integrals occurring in the later analysis. As ${ }_{n} \phi$ and ${ }_{n} \zeta$ are real, we have

$$
\begin{aligned}
& { }_{n} \phi_{\mathrm{k}}={ }_{n} \phi_{-\mathrm{k}}^{*}, \\
& { }_{n} Z_{\mathrm{k}}={ }_{n} Z_{-\mathrm{k}}^{*} .
\end{aligned}
$$

Substituting (1.18) and (1.19) into (1.12) and (1.13), we obtain the general differential equations for the perturbation amplitudes ${ }_{n} Z_{\mathbf{k}}$ and ${ }_{n} \phi_{\mathbf{k}}$ :

$$
\begin{aligned}
& g_{n} Z_{\mathbf{k}}+\frac{\partial_{n}^{\prime} \phi_{\mathbf{k}}}{\partial t}=-\sum_{v} \sum_{\mathbf{k}_{1}+\mathbf{k}_{2}+\ldots+\mathbf{k}_{p}=\mathbf{k}}\left\{\frac{\partial^{p-1}\left[\cosh \left(k_{p} h\right)\right] / \partial h^{p-1}}{(p-1) ! \cosh \left(k_{p} h\right)}{ }_{{ }_{1}} Z_{\mathbf{k}_{1} \nu_{\mathbf{2}}} Z_{\mathbf{k}_{\mathbf{z}} \cdots v_{p-2}} Z_{\mathbf{k}_{p-1}} \frac{\partial}{\partial t^{v_{p}}} \phi_{\mathbf{k}_{p}}\right. \\
& +\frac{\nu_{1} Z_{\mathbf{k}_{1} \nu_{2}} Z_{\mathbf{k}_{2}} \cdots_{\nu_{p-2}} Z_{\mathbf{k}_{p-2} \nu_{p-1}} \phi_{\mathbf{k}_{p-1} \nu_{p}} \phi_{\mathbf{k}_{p}}}{2(p-2) ! \cosh \left(k_{p-1} h\right) \cosh \left(k_{p} h\right)}\left[k_{p-1} k_{p} \frac{\partial^{p-2}}{\partial h^{p-2}}\left[\sinh \left(k_{p-1} h\right) \sinh \left(k_{p} h\right)\right]\right. \\
& \left.\left.-\left(\mathbf{k}_{p-1} \cdot \mathbf{k}_{p}\right) \frac{\partial^{p-2}}{\partial h^{p-2}}\left[\cosh \left(k_{p-1} h\right) \cosh \left(k_{p} h\right)\right]\right]\right\}
\end{aligned}
$$




$$
\begin{aligned}
& \frac{\partial^{2}}{\partial t^{2}} \phi_{\mathbf{k}}+\omega_{\mathbf{k} n}^{2} \phi_{\mathbf{k}}=-\sum_{\nu} \sum_{\mathbf{k}_{1}+\mathbf{k}_{2}+\ldots+\mathbf{k}_{p}=\mathbf{k}}\left\{\frac { \partial } { \partial t } \left(\frac{\nu_{1} Z_{\mathbf{k}_{1} \nu_{2}} Z_{\mathbf{k}_{2}} \cdots_{\nu_{p-1}} Z_{\mathbf{k}_{p-1}}}{(p-1) ! \cosh \left(k_{p} h\right)} \frac{\partial^{p-1}}{\partial h^{p-1}} \cosh \left(k_{p} h\right) \frac{\partial}{\partial t^{\nu_{p}}} \phi_{\mathbf{k}_{p}}\right.\right. \\
& +\frac{{ }_{1} Z_{\mathbf{k}_{1} \nu_{2}} Z_{\mathbf{k}_{2}} \cdots \nu_{p-2} Z_{\mathbf{k}_{p-2} \nu_{p-1}} \phi_{\mathbf{k}_{p-1} \nu_{p}} \phi_{\mathbf{k}_{p}}}{2(p-2) ! \cosh \left(k_{p-1} h\right) \cosh \left(k_{p} h\right)}\left[k_{p-1} k_{p} \frac{\partial^{p-2}}{\partial h^{p-2}}\left[\sinh \left(k_{p-1} h\right) \sinh \left(k_{p} h\right)\right]\right. \\
& \left.\left.-\left(\mathbf{k}_{p-1} \cdot \mathbf{k}_{p}\right) \frac{\partial^{p-2}}{\partial h^{p-2}}\left[\cosh \left(k_{p-1} h\right) \cosh \left(k_{p} h\right)\right]\right]\right) \\
& +g \frac{{ }_{\nu_{1}} Z_{\mathbf{k}_{1} \nu_{2}} Z_{\mathbf{k}_{2} \cdots \nu_{p-1}} Z_{\mathbf{k}_{p-1} \nu_{p}} \phi_{\mathbf{k}_{p}}}{(p-1) ! \cosh \left(k_{p} h\right)} \frac{\partial^{p}}{\partial h^{p}} \cosh \left(k_{p} h\right) \\
& \left.+g \frac{\nu_{\nu_{1}} Z_{\mathbf{k}_{1} \nu_{2}} Z_{\mathbf{k}_{2}} \cdots \nu_{p-1} Z_{\mathbf{k}_{p-1} \nu_{p}} \phi_{\mathbf{k}_{p}}}{(p-2) ! \cosh \left(k_{p} h\right)}\left(\mathbf{k}_{p-1} \cdot \mathbf{k}_{p}\right) \frac{\partial^{p-2}}{\partial h^{p-2}} \cosh \left(k_{p} h\right)\right\} \\
& \omega_{\mathrm{k}}=\sqrt{ }\{g k \tanh (k h)\} \text {. }
\end{aligned}
$$

For $n=1,(1.22)$ and (1.23) have the well-known linear solutions

$$
\begin{aligned}
& { }_{1} \phi_{\mathbf{k}}(t)={ }_{1} \phi_{\mathbf{k}}^{-} e^{i \omega_{\mathbf{k}} t}+{ }_{1} \phi_{\mathbf{k}}^{+} e^{-i \omega_{\mathbf{k}} t} \\
& { }_{1} Z_{\mathbf{k}}(t)={ }_{1} Z_{\mathbf{k}} e^{i \omega_{\mathbf{k}} t}+{ }_{1} Z_{\mathbf{k}}^{+} e^{-i \omega_{\mathbf{k}} t}
\end{aligned}
$$

with

$$
{ }_{1} Z_{\mathrm{k}}^{ \pm}= \pm \frac{i \omega_{\mathbf{k}}}{g}{ }_{1} \phi_{\mathrm{k}}^{ \pm} \quad\left({ }_{1} \phi_{\mathrm{k}}^{ \pm} \text {constant }\right)
$$

and, on account of (1.20) and (1.21),

$$
\begin{aligned}
& { }_{1} \phi_{\mathbf{k}}^{+}=\left({ }_{1} \phi_{-\mathbf{k}}^{-}\right)^{*}, \\
& { }_{1} Z_{\mathbf{k}}^{+}=\left({ }_{1} Z_{-\mathbf{k}}^{-}\right)^{*} .
\end{aligned}
$$

The sign indices refer to the direction of propagation of the waves relative to the wave-number direction. The wave amplitudes ${ }_{1} \phi_{\mathrm{z}}^{ \pm}$are determined by the initial conditions (1.14) and (1.15). Assuming the perturbation solutions of order lower than $n$ already expressed in terms of the linear solutions, the differential equation (1.23) for ${ }_{n} \phi_{\mathrm{k}}$ will then have the general form

$$
\frac{d^{2}}{d t^{2}}{ }_{n} \phi_{\mathrm{k}}+\omega_{\mathbf{z} n}^{2} \phi_{\mathrm{k}}=\sum_{\substack{\mathbf{k}_{1}+\mathbf{k}_{2}+\ldots+\mathbf{k}_{n}=\mathbf{k} \\ s_{1}, s_{2}, \ldots, s_{n}}} A_{\mathbf{k}_{1}, \mathbf{k}_{2}, \ldots, \mathbf{k}_{n}}^{s_{1,}, s_{2}, \ldots, s_{n}}(t)_{1} \phi_{\mathbf{k}_{1} 1}^{s_{1}} \phi_{\mathbf{k}_{2}}^{s_{2}} \ldots{ }_{1} \phi_{\mathbf{k}_{n}}^{s_{n}},
$$

with initial conditions

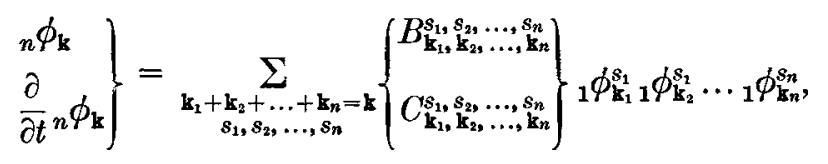

where the $s_{j}$ denote sign indices.

From some order $n$ onwards we shall now expect equation (1.29) to have nonstationary (resonant) solutions which grow continually with time, representing finally an appreciable modification of the initial sea state. The aim of our investigation will then be to determine this change in the sea state. At first sight, a basic difficulty appears to lie in our method of approach, which ceases to be applicable just at the point where the perturbation solutions begin to become of interest, i.e. for perturbations comparable in magnitude to the linear solution. 
This difficulty is overcome by deriving from our perturbation analysis an integro-differential equation for the rate of change of the sea state, the sea state for finite times after the initial time then being obtained by integration.

At this point we introduce the basic assumption that in the linear approximation the initial sea state is not only homogeneous, but also stationary and Gaussian. This implies that the first-order amplitudes are statistically independent for different wave-numbers and different directions of propagation. (The assumption is almost, but not quite, equivalent to assuming the initial functions $\zeta_{0}$ and $\partial \phi_{0} / \partial \eta$ to be Gaussian. In this case the first-order amplitudes are statistically independent for different wave-numbers, but not necessarily for opposite directions of propagation of the same wave-number, i.e. the sea is not necessarily stationary.) The Gaussian property of the sea follows from a loose application of the Central Limit Theorem provided the time of development of the sea is large in comparison to the periods occurring in the spectrum (which is a necessary condition for describing the sea as a quasi-homogeneous, quasi-stationary process in any case).

As a Gaussian sea is described completely by its two-dimensional energy spectrum, our aim will thus be to determine the change in the initial energy spectrum brought about by the non-steady higher-order perturbations. As a first step we shall then obviously need to determine the energy of the sea in terms of the perturbation functions considered above.

\section{The energy of the sea}

The mean energy of the sea per unit projection area is

$$
E=E_{\mathrm{kin}}+E_{\mathrm{pot}}=\frac{1}{2} \int_{-h}^{\zeta} \rho(\nabla \phi)^{2} d z+\frac{1}{2} \rho g \overline{\zeta^{2}},
$$

where the bars denote ensemble means. Using the Gauss formula and (1.1), (2.1) can be written in the form

$$
E=\frac{1}{2} \rho\left\{\overline{\left.\phi\left(\frac{\partial \phi}{\partial z}-\tilde{\nabla} \phi . \tilde{\nabla} \zeta\right)\right\}_{z=\zeta}}+\frac{1}{2} \rho g \overline{\zeta^{2}}\right.
$$

or, developing the first term about $z=0$,

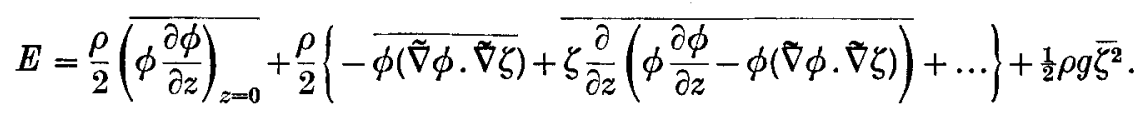

If $E$ is now developed in a perturbation series

$$
E={ }_{2} E+{ }_{3} E+{ }_{4} E+\ldots,
$$

we can obtain the terms ${ }_{n} E$ by substituting the perturbation and Fourier developments of $\phi$ and $\zeta$ into (2.3). The first term is then determined by the linear solution

or, in integral representation,

$$
{ }_{2} E=\frac{2 \rho}{g} \sum_{\mathbf{k}} \omega_{\mathbf{k}}^{2} \overline{\left.\Phi_{1} \Phi_{\mathbf{k}}^{+}\right|^{2}}
$$

$$
{ }_{2} E=\iint_{-\infty}^{+\infty} F(\mathbf{k}) d k_{x} d k_{y}
$$


where $_{2} F(\mathbf{k})$ is the two-dimensional energy spectrum (in the linear approximation), defined in the sense that only waves travelling in the positive $\mathbf{k}$-direction contribute to the spectrum at $\mathbf{k}$.

The perturbation terms of odd order depend only on odd mean products of the first-order perturbation amplitudes. For a Gaussian sea these vanish:

$$
{ }_{3} E={ }_{5} E={ }_{7} E=\ldots=0 .
$$

For the next two even-order terms we find

$$
\begin{aligned}
& { }_{4} E=\frac{\rho \omega_{\mathbf{k}}^{2}}{2 g} \sum_{\mathbf{k}}\left\{\overline{\left.{ }_{2} \Phi_{\mathbf{k}}\right|^{2}}+2 \operatorname{Re}\left(\overline{{ }_{1} \Phi_{-\mathbf{k} 3} \Phi_{\mathbf{k}}}\right)\right\}+\frac{1}{2} \rho g \sum_{\mathbf{k}}\left\{\overline{\left|{ }_{2} Z_{\mathbf{k}}\right|^{2}}+2 \operatorname{Re}\left(\overline{{ }_{1} Z_{-\mathbf{k} 3} Z_{\mathbf{k}}}\right)\right\}+\Sigma \ldots \\
& { }_{6} E=\frac{\rho \omega_{\mathrm{k}}^{2}}{2 g} \sum_{\mathbf{k}}\left\{\overline{\left.{ }_{3} \Phi_{\mathrm{k}}\right|^{2}}+2 \operatorname{Re}\left(\overline{\Phi \Phi_{-\mathrm{k} 4} \Phi_{\mathrm{k}}}\right)+2 \operatorname{Re}\left(\overline{\Phi_{1} \Phi_{-\mathrm{k} 5} \Phi_{\mathrm{k}}}\right)\right\} \\
& +\frac{1}{2} \rho g \sum_{\mathbf{k}}\left\{\left|{ }_{3} Z_{\mathbf{k}}\right|^{2}+2 \operatorname{Re}\left(\overline{{ }_{2} Z_{-\mathbf{k} 4} Z_{k}}\right)+2 \operatorname{Re}\left(\overline{{ }_{1} Z_{-\mathbf{k} 5} Z_{\mathbf{k}}}\right)\right\}+\Sigma \ldots
\end{aligned}
$$

The indicated additional sums contain mean products of three or more perturbation amplitudes. It will be shown later that these remain constant and therefore have no influence in the present problem. We shall find further that ${ }_{4} E$ also contains only constant terms, so that the energy transfer is finally determined by the terms in the first two sums in ${ }_{6} E$. We note that, on account of (1.22), corresponding terms in the two sums would be equal, just as in the linear approximation, if the ${ }_{n} \Phi_{\mathbf{k}}$ were periodic with their natural frequency $\omega_{\mathbf{k}}$ (except for additional terms entering in the third sum). We shall find later that the nonstationary contributions to ${ }_{6} E$ arise solely from such free-wave components, so that it will suffice then to investigate only the first sum in (2.9). The problem thus reduces to the analysis of the covariance products in (2.9), which involve all perturbation amplitudes ${ }_{n} \Phi_{\mathbf{k}}$ from the first to the fifth orders. This is essentially a statistical problem, the solution of which is based on certain asymptotic integral formulae which will be considered in the next section.

\section{The asymptotic response of an undamped oscillator to stationary and non-stationary random excitations}

Let $\mathscr{F}_{1}\left(\omega, \omega^{\prime} ; t\right)$ be the solution of the differential equation

$$
\frac{d^{2} \psi}{d t^{2}}+\omega^{2} \psi=\exp \left(i \omega^{\prime} t\right)
$$

with the initial values $\psi=d \psi / d t=0$ for $t=0$. We have

$$
\mathscr{I}_{1}\left(\omega, \omega^{\prime} ; t\right)=\left\{\begin{array}{l}
\frac{e^{i \omega^{\prime} t}}{\omega^{2}-\omega^{\prime 2}}-\frac{1}{2 \omega}\left(\frac{e^{i \omega t}}{\omega-\omega^{\prime}}+\frac{e^{-i \omega t}}{\omega+\omega^{\prime}}\right) \text { for } \omega^{\prime 2} \neq \omega^{2}, \\
\frac{t e^{i \omega^{\prime} t}}{2 i \omega^{\prime}}+\frac{e^{i \omega t}-e^{-i \omega t}}{4 \omega \omega^{\prime}} \text { for } \omega^{\prime 2}=\omega^{2}
\end{array}\right.
$$

If $g(\omega)$ is an arbitrary continuous real function it can then be shown that

$$
\lim _{t \rightarrow \infty} \frac{d}{d t} \int_{-\infty}^{+\infty} \mathscr{I}_{1}\left(\omega, \omega^{\prime} ; t\right) \mathscr{I}_{1}\left(\omega,-\omega^{\prime} ; t\right) g\left(\omega^{\prime}\right) d \omega^{\prime}=\frac{\pi}{2 \omega^{2}}\{g(\omega)+g(-\omega)\},
$$


or, in terms of Dirac functions,

$$
\lim _{t \rightarrow \infty} \frac{d}{d t}\left\{\mathscr{I}_{1}\left(\omega, \omega^{\prime} ; t\right) \mathscr{I}_{1}\left(\omega,-\omega^{\prime} ; t\right)\right\}=\frac{\pi}{2 \omega^{2}}\left\{\delta\left(\omega+\omega^{\prime}\right)+\delta\left(\omega-\omega^{\prime}\right)\right\}
$$

It can be shown further that

$$
\lim _{t \rightarrow \infty} \frac{d}{d t} \int_{-\infty}^{+\infty} \mathscr{I}_{1}\left(\omega, \omega^{\prime} ; t\right) e^{-i \omega^{\prime} l} g\left(\omega^{\prime}\right) d \omega^{\prime}=0
$$

We shall use (3.6) later in the more general form

$$
\lim _{t \rightarrow \infty} \frac{d}{d t} \int \ldots \int \mathscr{I}_{1}\left(\omega, \omega^{\prime} ; t\right) e^{-i \omega^{\prime} t} g\left(\lambda_{1}, \lambda_{2}, \ldots, \lambda_{n}\right) d \lambda_{1} d \lambda_{2} \ldots d \lambda_{n}=0,
$$

with $\omega=\omega\left(\lambda_{1}, \lambda_{2}, \ldots, \lambda_{n}\right), \omega^{\prime}=\omega^{\prime}\left(\lambda_{1}, \lambda_{2}, \ldots, \lambda_{n}\right)$ and $\partial\left(\omega^{\prime} \pm \omega\right) / \partial n \neq 0$ on the surfaces $\omega^{\prime} \pm \omega=0$, where $n$ denotes the surface normal.

In particular, if $\psi_{1}$ is a solution of the differential equation

$$
\frac{d^{2} \psi}{d t^{2}}+\omega^{2} \psi=r(t)
$$

where $r(t)$ is a stationary (not necessarily real) random function with a continuous spectral density function $f(\omega)$, we find by applying (3.4) and (3.6) to the spectral representation of $\psi_{1}$

$$
\begin{array}{cc}
\lim _{t \rightarrow \infty} \frac{d}{d t} \overline{\left|\psi_{1}\right|^{2}}=\frac{\pi}{2 \omega^{2}}\{f(\omega)+f(-\omega)\}, \\
\text { and } \\
\lim _{t \rightarrow \infty} \frac{d}{d t} \overline{\left(\psi_{1} r^{*}\right)}=0 .
\end{array}
$$

An expression similar to (3.9), in which the spectral densities were expressed in terms of their correlation integrals, was derived by Phillips (1957). According to (3.9), the asymptotic response of an undamped oscillator to stationary random excitation is non-stationary, the mean-square amplitude increasing at a rate proportional to the spectral density of the excitation at the resonant frequency.

We consider further the solution $\mathscr{I}_{2}\left(\omega, \omega^{\prime}, \omega^{\prime \prime}, \omega^{\prime \prime \prime} ; t\right)$ of the differential equation

$$
\frac{d^{2} \psi}{d t^{2}}+\omega^{2} \psi=e^{i \omega^{\prime} t} \mathscr{I}_{1}\left(\omega^{\prime \prime}, \omega^{\prime \prime \prime} ; t\right)
$$

with the initial values $\psi=d \psi / d t=0$ for $t=0$. We have

$$
\mathscr{I}_{2}=\frac{\mathscr{I}_{1}\left(\omega^{\prime \prime}, \omega^{\prime \prime \prime} ; t\right) e^{i \omega^{\prime} t}}{\omega^{2}-\left(\omega^{\prime}+\omega^{i \prime \prime}\right)^{2}}+\ldots \quad \text { for } \quad\left\{\begin{array}{l}
\left(\omega^{\prime}+\omega^{\prime \prime \prime}\right)^{2} \\
\left(\omega^{\prime} \pm \omega^{\prime \prime}\right)^{2}
\end{array}\right\} \neq \omega^{2},
$$

where the indicated additional terms are steady oscillations which remain bounded in the case of resonance in $\mathscr{I}_{1}$, i.e. for $\omega^{\prime \prime 2}=\omega^{\prime \prime \prime 2}$.

In the case of resonance in both $\mathscr{I}_{2}$ and $\mathscr{I}_{1}$, we find

$$
\mathscr{I}_{2}=-\frac{t^{2} \exp \left[i\left(\omega^{\prime}+\omega^{\prime \prime \prime}\right) t\right]}{8 \omega^{\prime \prime \prime}\left(\omega^{\prime}+\omega^{\prime \prime \prime}\right)}-\frac{i t\left(2 \omega^{\prime \prime \prime}+\omega^{\prime}\right)}{\omega^{\prime \prime \prime}\left(\omega^{\prime}+\omega^{\prime \prime \prime}\right)^{2}} \exp \left[i\left(\omega^{\prime}+\omega^{\prime \prime \prime}\right) t\right]+\ldots
$$

for $\omega^{\prime \prime \prime 2}=\omega^{\prime \prime 2},\left(\omega^{\prime}+\omega^{\prime \prime \prime}\right)^{2}=\omega^{2}$, where the additional terms are again bounded steady oscillations. 
Integral expressions similar to (3.4) and (3.6) can be considered generally for the solution $\mathscr{F}_{2}$, yielding information on the asymptotic response of an undamped oscillator to forcing functions which can be represented as the product of a stationary random function and a non-stationary random oscillation of the type $\psi_{1}$. For the later analysis, however, we shall require only the relation

$$
\begin{aligned}
\lim _{t \rightarrow \infty} \frac{d}{d t}\left\{\operatorname{Re}\left[e^{-i \omega t} \mathscr{I}_{2}\left(\omega_{1}-\omega^{\prime}, \omega^{\prime \prime}, \omega+\omega^{\prime} ; t\right)\right]\right\} \\
=-\frac{\pi}{4 \omega\left(\omega+\omega^{\prime}\right)}\left\{\delta\left(\omega+\omega^{\prime}+\omega^{\prime \prime}\right)+\delta\left(\omega+\omega^{\prime}-\omega^{\prime \prime}\right)\right\} .
\end{aligned}
$$

The derivation of the asymptotic relations (3.5), (3.6) and (3.14) will not be given. We remark only that the $\delta$-functions enter on account of the rapid oscillation of the exponential terms, which cause all contributions to the integrals to vanish for large $t$ except in the neighbourhood of the poles of the integrands. The contributions from the poles can then be evaluated by contour integration in the complex frequency plane.

\section{The energy transfer}

We investigate first the energy perturbation terms ${ }_{4} E$ and ${ }_{6} E$, applying the results of the previous section.

\section{(a) The term ${ }_{4} E$}

The term ${ }_{4} E$ depends on the first three perturbation amplitudes. For the secondorder amplitude ${ }_{2} \Phi_{\mathbf{k}}$ we find from (1.22), (1.23)

$$
\begin{aligned}
& \frac{d^{2}}{d t^{2}}{ }_{2} \Phi_{\mathbf{k}}+\omega_{\mathbf{k} 2}^{2} \Phi_{\mathbf{k}}=\sum_{\substack{\mathbf{k}_{1}+\mathbf{k}_{2}=\mathbf{k} \\
s_{1}, s_{2}}} D_{\mathbf{l}_{1}, \mathbf{k}_{2} 1}^{s_{1}, s_{2}} \Phi_{\mathbf{k}_{1} 1}^{s_{1}} \Phi_{\mathbf{l}_{2}}^{s_{2}} \exp \left[-i\left(s_{1} \omega_{\mathbf{k}_{1}}+s_{2} \omega_{\mathbf{k}_{2}}\right) t\right], \\
& { }_{2} Z_{\mathbf{k}}=-\frac{1}{g} \frac{d}{d t}{ }_{2} \Phi_{\mathbf{k}}+\sum_{\substack{\mathbf{k}_{1}+\mathbf{k}_{2}=\mathbf{k} \\
s_{1}, s_{2}}} E_{\mathbf{k}_{1}, \mathbf{k}_{2} 1}^{s_{1}, s_{2}} \Phi_{\mathbf{k}_{1} 1}^{s_{1}} \Phi_{\mathbf{k}_{2}}^{s_{2}} \exp \left[-i\left(s_{1} \omega_{\mathbf{k}_{1}}+s_{2} \omega_{\mathbf{k}_{2}}\right) t\right],
\end{aligned}
$$

with

$$
\begin{aligned}
& D_{\mathbf{k}_{1}, \mathbf{k}_{2}}^{s_{1}, s_{2}}=i\left(\omega_{1}+\omega_{2}\right)\left(k_{1} k_{2} \tanh k_{1} h \tanh k_{2} h-\left(\mathbf{k}_{1} \cdot \mathbf{k}_{2}\right)\right)-\frac{i}{2}\left(\frac{\omega_{1} k_{2}^{2}}{\cosh ^{2} k_{2} h}+\frac{\omega_{2} k_{1}^{2}}{\cosh ^{2} k_{1} h}\right) \\
& E_{\mathbf{k}_{1}, \mathbf{k}_{2}}^{s_{1}, s_{2}}=\frac{1}{2 g}\left\{\left(\mathbf{k}_{1}, \mathbf{k}_{2}\right)-g^{-2} \omega_{1} \omega_{2}\left(\omega_{1}^{2}+\omega_{2}^{2}+\omega_{1} \omega_{2}\right)\right\}
\end{aligned}
$$

where the abbreviation $\omega_{j}=s_{j} \omega_{\mathbf{k}_{j}}$ has been introduced.

The terms in the sum (4.1) excite steady oscillations in ${ }_{2} \Phi_{\mathbf{k}}$ if $\left(s_{1} \omega_{\mathbf{k}_{1}}+s_{2} \omega_{\mathbf{k}_{3}}\right)^{2} \neq \omega_{\mathrm{k}}^{2}$ and non-steady resonant oscillations if $\left(s_{1} \omega_{\mathbf{k}_{1}}+s_{2} \omega_{\mathbf{k}_{2}}\right)^{2}=\omega_{\mathbf{k}}^{2}$. For finite-depth gravity waves the natural frequency curve $\omega_{\mathbf{k}}=(g k \tanh k h)^{\frac{1}{2}}=\Omega(k)$ passes through the origin and is convex for $k>0$. From these properties it follows that

$$
\Omega\left(k_{1}+k_{2}\right) \leqslant \Omega\left(k_{1}\right)+\Omega\left(k_{2}\right) .
$$

As $\Omega(k)$ is also monotonic, the inequality

yields further

$$
\begin{gathered}
\left|\mathbf{k}_{1}+\mathbf{k}_{2}\right| \leqslant k_{1}+k_{2} \\
\Omega\left(\left|\mathbf{k}_{1}+\mathbf{k}_{2}\right|\right) \leqslant \Omega\left(k_{1}+k_{2}\right) .
\end{gathered}
$$


Combining both inequalities, we have

$$
\omega_{\mathbf{k}_{1}+\mathbf{k}_{3}} \leqslant \omega_{\mathbf{k}_{\mathbf{1}}}+\omega_{\mathbf{k}_{\mathbf{2}}}
$$

The substitution $\mathbf{k}^{\prime}=\mathbf{k}_{1}+\mathbf{k}_{2}, \mathbf{k}^{\prime \prime}=-\mathbf{k}_{2}$ then yields

so that finally

$$
\begin{gathered}
\omega_{\mathbf{k}^{\prime}}-\omega_{\mathbf{k}^{\prime \prime}} \leqslant \omega_{\mathbf{k}^{\prime}+\mathbf{k}^{\prime \prime}}, \\
\omega_{\mathbf{k}_{1}}-\omega_{\mathbf{k}_{\mathbf{a}}} \leqslant \omega_{\mathbf{k}_{1}+\mathbf{k}_{2}}\left(=\omega_{\mathbf{k}}\right) \leqslant \omega_{\mathbf{k}_{1}}+\omega_{\mathbf{k}_{2}} .
\end{gathered}
$$

The equality sign on the left holds only for $\mathbf{k}_{\mathbf{2}}=0$ or $\mathbf{k}_{\mathbf{1}}+\mathbf{k}_{\mathbf{2}}=0$, the equality sign on the right only for $\mathbf{k}_{\mathbf{1}}=0$ or $\mathbf{k}_{\mathbf{2}}=0$. For these cases $D_{\mathbf{k}_{1}, \mathbf{k}_{\mathbf{2}}}^{s_{1}, s_{2}}$ vanishes. Hence none of the exciting terms in (4.1) satisfy the resonance condition $\left(s_{1} \omega_{\mathbf{k}_{1}}+s_{2} \omega_{\mathbf{k}_{2}}\right)^{2}=\omega_{\mathbf{k}}^{2}$, so that the solution ${ }_{2} \Phi_{\mathbf{k}}$ is composed entirely of steady harmonic oscillations

$$
\begin{aligned}
& { }_{2} \Phi_{\mathbf{k}}=\sum_{\substack{\mathbf{k}_{1}+\mathbf{k}_{2}=\mathbf{k} \\
s_{1}, s_{2}}} \frac{D_{\mathbf{k}}-\left(s_{1} \omega_{\mathbf{k}_{1}}+s_{\mathbf{k}_{2}}+s_{\mathbf{2}} \omega_{\mathbf{k}_{2}}\right)^{2}}{s_{1}} \Phi_{\mathbf{k}_{1} \mathbf{1}}^{s_{1}} \Phi_{\mathbf{l}_{\mathbf{2}}}^{s_{\mathbf{z}_{2}}} \exp \left[-i\left(s_{1} \omega_{\mathbf{k}_{1}}+s_{\mathbf{2}} \omega_{\mathbf{k}_{\mathbf{2}}}\right) t\right] \\
& +\sum_{\substack{\mathbf{k}_{1}+\mathbf{k}_{2}=\mathbf{k} \\
\mathbf{s}_{1}, s_{2}}} \Phi_{\mathbf{k}_{1} 1}^{s_{1}} \Phi_{\mathbf{k}_{2}}^{s_{2}}\left\{F_{\mathbf{k}_{1}, \mathbf{k}_{2}}^{s_{1}, s_{2}} e^{i \omega_{\mathbf{k}} l}+G_{\mathbf{k}_{2}, \mathbf{k}_{2}}^{s_{1}, s_{2}} e^{-i \omega_{\mathbf{k}} t}\right\}
\end{aligned}
$$

The free oscillations in the second sum are determined by the initial conditions. We shall expect, intuitively, initial conditions of order higher than the first to have no influence for our problem, the rate of change of the sea state being determined (to the first contributing order) only by forced oscillations of the type in the first sum. On the other hand, it is not immediately evident mathematically that lower-order free oscillations do not generate non-steady resonant oscillations in the higher-order perturbation solutions which are comparable in their influence on the sea state to the resonant oscillations generated by the forced lower-order oscillations. We shall, none the less, neglect all free oscillation terms depending on initial values of higher order than the first in the following, mainly because the number of these terms increases so rapidly with the perturbation order that the equations soon become extremely complicated if all are included. It is not difficult to see $a$ posteriori that the terms do, in fact, leave the final result unchanged.

Since ${ }_{2} \Phi_{k}$ contains only steady oscillations, the term $\overline{\left.{ }_{2} \Phi_{k}\right|^{2}}$ in the first sum in (2.8) remains constant. From (1.22), the term $\overline{\left.{ }_{2} Z_{\mathbf{k}}\right|^{2}}$ in the second sum then also remains constant. The indicated second sum contains mean products of three and more perturbation amplitudes and hence cannot contain perturbation amplitudes of higher order than the second. The terms in this sum are therefore also stationary. There remains the term $2 \operatorname{Re}\left(\overline{\left.{ }_{1} \Phi_{-\mathbf{k} 3} \Phi_{\mathbf{k}}\right)}\right.$ in the first sum and

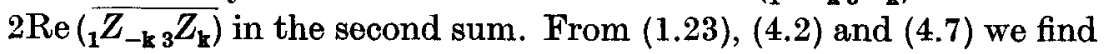

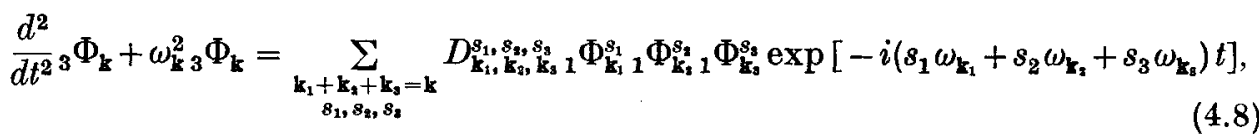

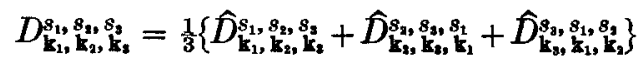


with

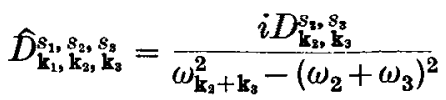

$$
\begin{aligned}
& \times\left\{2\left(\omega_{1}+\omega_{2}+\omega_{3}\right)\left(\frac{\omega_{1}^{2} \omega_{\mathbf{k}_{2}+\mathbf{k}_{2}}^{2}}{g^{2}}-\mathbf{k}_{1} \cdot\left(\mathbf{k}_{2}+\mathbf{k}_{3}\right)\right)-\frac{\omega_{1}\left(\mathbf{k}_{2}+\mathbf{k}_{3}\right)^{2}}{\cosh ^{2}\left(\left|\mathbf{k}_{2}+\mathbf{k}_{3}\right| h\right)}\right\} \\
& -i D_{\mathbf{k}_{2}, \mathbf{k}_{3}}^{s_{3}, s_{3}} \frac{\omega_{1}}{g^{2}}\left(\omega_{1}^{2}+\omega_{\mathbf{k}_{2}}^{2}+\mathbf{k}_{3}\right)+E_{\mathbf{k}_{3}, \mathbf{k}_{3}}^{s_{2}, s_{3}}\left\{\frac{\omega_{1}^{3}\left(\omega_{2}+\omega_{3}\right)}{g}-g \mathbf{k}_{1} \cdot\left(\mathbf{k}_{2}+\mathbf{k}_{3}\right)\right\} \\
& +\frac{\omega_{1}}{2 g^{2}}\left(\mathbf{k}_{2} \cdot \mathbf{k}_{3}\right)\left\{\left(\omega_{1}+\omega_{2}+\omega_{3}\right)\left(\omega_{2}^{2}+\omega_{3}^{2}\right)+\omega_{2} \omega_{3}\left(\omega_{2}+\omega_{3}\right)\right\} \\
& -\frac{\omega_{1} \omega_{2}^{2} k_{3}^{2}}{2 g^{2}}\left(\omega_{1}+\omega_{2}+2 \omega_{3}\right)-\frac{\omega_{1} \omega_{3}^{2} k_{2}^{2}}{2 g^{2}}\left(\omega_{1}+2 \omega_{2}+\omega_{3}\right),
\end{aligned}
$$

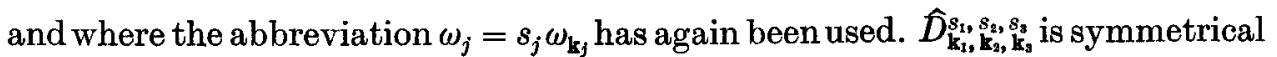

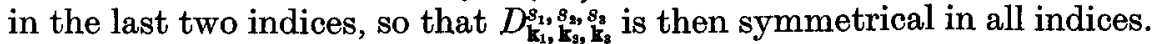

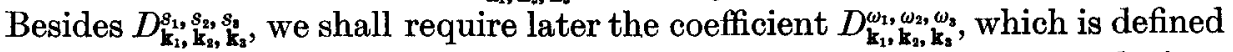
formally in the same way as $D_{\mathbf{l}_{1}, k_{s}, \mathbf{k}_{3}}^{s_{1} s_{2}, s_{3}}$ except that in place of the linear solutions ${ }_{1} \Phi_{\mathbf{k}_{j}}^{s_{j}} \exp \left(-i s_{j} \omega_{\mathbf{k}_{j}} t\right)$ the general expressions $\Phi_{\mathbf{k}_{j}} \exp \left(-i \omega_{j} t\right)$ are substituted in (1.23), the frequencies $\omega_{j}$ being treated as independent variables. $\left(D_{\mathbf{k}_{1}, \mathbf{k}_{3}, \mathbf{k}_{z}}^{\omega_{1}, \omega_{3}, \omega_{3}}\right.$ differs from $D_{\mathbf{k}_{1},}^{s_{1}, s_{2}, s_{8}, s_{8}}$ as expressed in terms of $\omega_{j}=s_{j} \omega_{\mathbf{k}_{j}}$ in $(4.9)$ and $(4.10)$, since in these expressions $\omega_{j}$ is not independent of $\mathbf{k}_{\boldsymbol{j}}$, and free use of the identity

$$
\omega_{j}^{2}=g k_{j} \tanh k_{j} h
$$

has been made in simplifying (4.10).) The coefficient $D_{\mathbf{k}_{1}, \mathbf{k}_{2}, \mathbf{k}_{8}}^{\omega_{1}, \omega_{3}}$ does not enter into the final equation and thus need not be given explicitly.

The third-order perturbation solution ${ }_{3} \Phi_{k}$ is the first to contain non-steady resonant components, as it can readily be verified that with three frequencies the resonance condition $\left(s_{1} \omega_{\mathbf{k}_{1}}+s_{2} \omega_{\mathbf{k}_{3}}+s_{3} \omega_{\mathbf{k}_{3}}\right)^{2}=\omega_{\mathbf{k}}^{2}$ can be satisfied with a suitable choice of $\mathbf{k}_{j}$ and $s_{j}$. None the less, we shall find that the term

$$
2 \operatorname{Re}\left(\overline{{ }_{1} \Phi_{-153} \Phi_{k}}\right)
$$

in ${ }_{4} E$ remains constant, as the non-steady components of ${ }_{3} \Phi_{\mathbf{z}}$ are exactly $90^{\circ}$ out of phase with the corresponding components of ${ }_{1} \Phi_{k}$. For, from the Gaussian property of the sea, it follows that two linear amplitudes ${ }_{1} \Phi_{\mathbf{k}_{1}}^{s_{1}},{ }_{1} \Phi_{\mathbf{k}_{2}}^{s_{2}}$ are statistically independent unless they are a conjugate pair, with $\left(\begin{array}{c}s_{1} \\ \mathbf{k}_{1}\end{array}\right)=\left(\begin{array}{l}-s_{1} \\ -\mathbf{k}_{1}\end{array}\right)$. Making use of this property and allowing for the symmetry of $D_{\mathbf{l}_{1}, \mathbf{S}_{\mathbf{3}}, \mathbf{k}_{\mathbf{3}}}^{\boldsymbol{s}_{1}, \mathbf{s}_{2}, \mathbf{s}_{\mathbf{2}}}$, we find

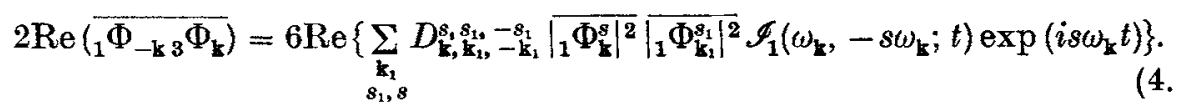

According to (3.3), the non-steady component of the resonant oscillation $\mathscr{I}_{1}\left(\omega_{\mathbf{k}},-s \omega_{\mathbf{k}} ; t\right)$ in $(4.11)$ is $90^{\circ}$ out of phase with the oscillation $e^{-i s \omega_{k} t}$. Since the coefficient $D_{\mathbf{l}_{1}, \mathbf{k}_{2}, \mathbf{k}_{6}}^{s_{1}}, \boldsymbol{s}_{2}, s_{\mathbf{2}}$ is real, the non-steady components therefore contribute only to the imaginary part of the sum in (4.11), and thus the term $2 \operatorname{Re}\left(\overline{{ }_{1} \Phi_{-\mathbf{k} 3} \overline{\Phi_{k}}}\right)$ in ${ }_{4} E$ also remains constant. In the same way, using $(1.22)$, it can be shown that $2 \operatorname{Re} \overline{\left(Z_{1} Z_{3} Z_{k}\right)}$ remains constant. 
The first perturbation term ${ }_{4} E$ thus represents a correction term arising from the steady higher-harmonic components, but not the non-linear energy transfer we are seeking for. The term has been analysed in greater detail by Tick (1958) (with the exception of the terms depending on the third-order perturbations).

The stationarity of ${ }_{4} E$ is a consequence of the steadiness of the second-order perturbations, which follows from the inequality (4.6). This, in turn, is in essence equivalent to the fact that for finite-depth gravity waves the group velocity is smaller than the phase velocity. In non-linear systems for which this is not the case, e.g. capillary waves, a continuous energy transfer would already occur in the term ${ }_{4} E$. Thus for capillary waves the non-linear energy transfer is probably considerably stronger than for gravity waves.

\section{(b) The term ${ }_{6} E$}

In the course of our analysis of the next term ${ }_{6} E$ we shall need to extend the perturbation analysis to the fifth order. With reference to the remark following equation (2.9), we investigate first only the sum containing the amplitudes of the potential perturbations. Allowing again for the statistical independence of the first-order amplitudes, we find for the first term in this sum depending only on the third-order perturbations

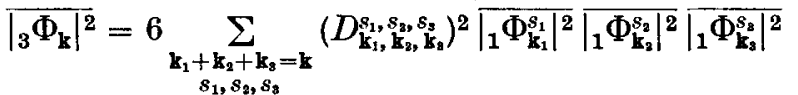

$$
\begin{aligned}
& \times \mathscr{I}_{1}\left(\omega_{\mathbf{k}}, s_{1} \omega_{\mathbf{k}_{1}}+s_{2} \omega_{\mathbf{k}_{3}}+s_{3} \omega_{\mathbf{k}_{3}} ; t\right) \mathscr{I}_{1}\left(\omega_{\mathbf{k}},-s_{1} \omega_{\mathbf{k}_{1}}-s_{2} \omega_{\mathbf{k}_{2}}-s_{3} \omega_{\mathbf{k}_{3}} ; t\right)
\end{aligned}
$$

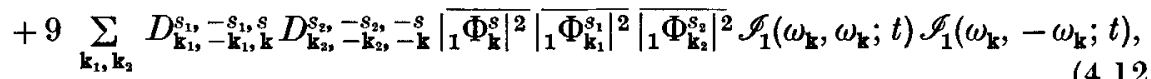

$$
\begin{aligned}
& s, s_{1}, s_{2}
\end{aligned}
$$

where use has been made of the symmetry of $D_{\mathbf{Z}_{1}, \mathbf{B}_{3}, \mathbf{Z}_{3}}^{s_{1}, s_{8}, s_{8}}$ in grouping the possible combinations of three conjugate index pairs occurring in the six first-order amplitudes involved in the mean product $\overline{\left.{ }_{3} \Phi_{\mathbf{k}}\right|^{2}}$.

According to (3.4), the first sum increases linearly in $t$ for large $t$, whereas from (3.3) the second sum increases quadratically. Hence the second sum will dominate ultimately. We shall find later, however, that the second sum cancels against a further term in 6 , so that only the first sum represents a genuine transfer term.

In both sums, the non-steady components are resonant oscillations with frequencies $\pm \omega_{\mathrm{k}}$. We can thus separate the non-steady terms in ${ }_{3} \Phi_{\mathrm{k}}$ into two components ${ }_{3} \Phi_{\mathbf{k}}^{+} e^{-i \omega_{\mathbf{k}} t}$ and ${ }_{3} \Phi_{\mathbf{k}}^{-} e^{i \omega_{\mathbf{k}} t}$, corresponding to the two directions of propagation relative to $\mathbf{k}$. If the components corresponding to different directions of propagation in (4.12) are then also separated, we obtain, applying (3.4) and (3.3),

where

$$
\overline{\left.\left.\right|_{3} \Phi_{\mathbf{k}}\right|^{2}}=\overline{\left.{ }_{3} \Phi_{\mathbf{k}}^{+}\right|^{2}}+\overline{\left.\left.\right|_{3} \Phi_{\mathbf{k}}^{-}\right|^{2}}+\text { stationary terms, }
$$

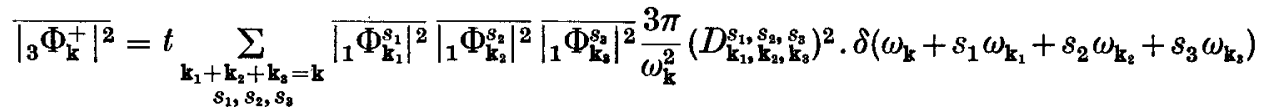

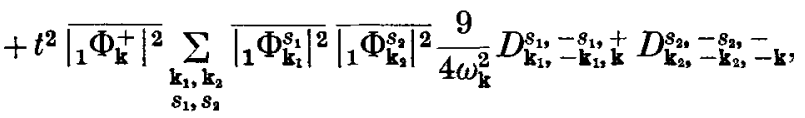

and

$$
\overline{\left.{ }_{3} \Phi_{\mathbf{k}}^{-}\right|^{2}}=\overline{\left.{ }_{3} \Phi_{-\mathbf{k}}^{+}\right|^{2}} \text {. }
$$


For the next term $2 \operatorname{Re}\left(\overline{\Phi_{2} \Phi_{4} \Phi_{k}}\right)$ in (2.9) we require the fourth-order amplitudes ${ }_{4} \Phi_{k}$. From (1.23) we find

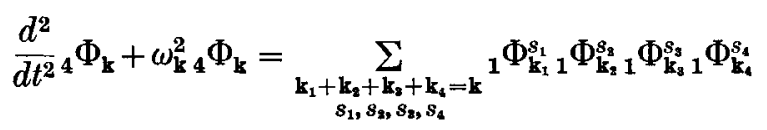

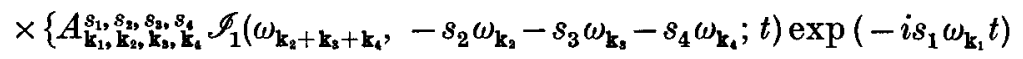

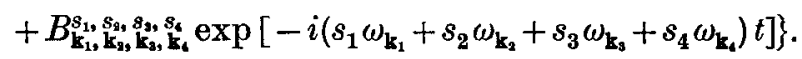

The first expression in the bracket is due to exciting terms which depend on non-steady third-order perturbations, whereas the second expression contains the remaining terms depending only on the steady first- and second-order perturbations. Of the coefficients we need to know only that they are imaginary

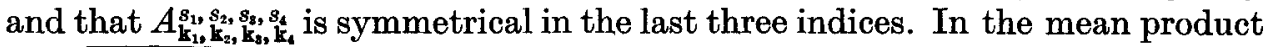
$2 \operatorname{Re}\left(\overline{2}_{2} \Phi_{-k 4} \Phi_{1 k}\right)$ we need again consider only those terms in which the six index groups $\left(\begin{array}{c}\mathbf{s}_{j} \\ \mathbf{k}_{j}\end{array}\right)$ involved form three conjugate pairs. Of the four index groups belonging to ${ }_{4} \Phi_{\mathrm{k}}$, two must then necessarily be a conjugate pair. Hence two of the frequencies in the exciting terms in (4.15) cancel, and it follows from the inequalities (4.6) that the remaining two frequencies can no longer satisfy the condition for resonant excitation of ${ }_{4} \Phi_{k}$. The second expression in (4.15) thus generates steady oscillations which, since ${ }_{2} \Phi_{-\mathrm{k}}$ is also stationary, yield only constant terms in $\operatorname{Re}\left(\overline{{ }_{2} \Phi_{-k 4} \Phi_{k}}\right)$ and need not be considered further. The first expression in (4.15) gives rise to non-steady oscillations in ${ }_{4} \Phi_{\mathbf{k}}$ if the factor $\mathscr{I}_{1}\left(\omega_{\mathbf{k}_{2}+\mathbf{k}_{3}+\mathbf{k}_{4}},-s_{2} \omega_{\mathbf{k}_{3}}-s_{3} \omega_{\mathbf{k}_{3}}-s_{4} \omega_{\mathbf{k}_{4}} ; t\right)$ is a resonant oscillation, but again no direct resonant excitation of ${ }_{4} \Phi_{k}$ takes place and we can apply (3.12) to express the solution simply in terms of $\mathscr{F}_{1}$. Allowing for the different combinations of conjugate index pairs we obtain

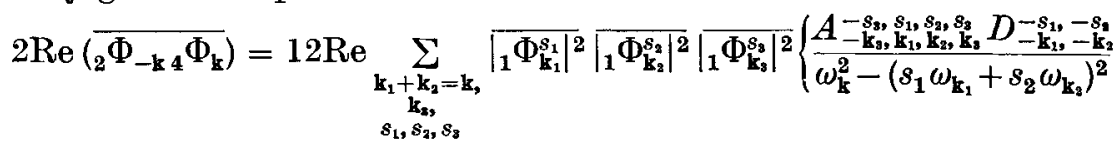

$$
\begin{aligned}
& \times \exp \left[i\left(s_{1} \omega_{\mathbf{k}_{1}}+s_{2} \omega_{\mathbf{k}_{2}}+s_{3} \omega_{\mathbf{k}_{3}}\right) t\right] \mathscr{I}_{1}\left(\omega_{\mathbf{k}_{1}+\mathbf{k}_{2}+\mathbf{k}_{2}},-s_{1} \omega_{\mathbf{k}_{1}}-s_{2} \omega_{\mathbf{k}_{8}}-s_{3} \omega_{\mathbf{k}_{3}} ; t\right)
\end{aligned}
$$

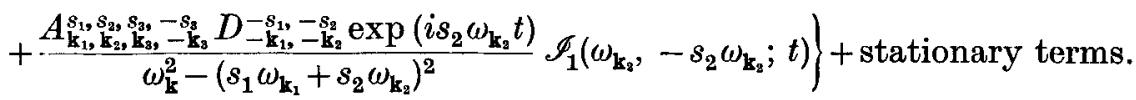

The oscillation $\mathscr{I}_{1}\left(\omega_{\mathbf{k}_{1}+\mathbf{k}_{2}+\mathbf{k}_{\mathbf{g}}},-s_{1} \omega_{\mathbf{k}_{1}}-s_{\mathbf{2}} \omega_{\mathbf{k}_{\mathbf{z}}}-s_{3} \omega_{\mathbf{k}_{\mathrm{g}}} ; t\right)$ in the first expression in (4.16) is non-steady for certain values of $\mathbf{k}_{j}$ and $s_{j}$. However, from (3.7) the sum (or, in reality, the integral) of the product of this oscillation with the steady oscillation $\exp \left[i\left(s_{1} \omega_{\mathbf{k}_{1}}+s_{\mathbf{2}} \omega_{\mathbf{k}_{2}}+s_{3} \omega_{\mathbf{k}_{\mathbf{3}}}\right) t\right]$ remains constant. The oscillation $\mathscr{F}_{1}\left(\omega_{\mathbf{k}_{2}},-s_{2} \omega_{\mathbf{k}_{3}} ; t\right)$ in the second expression is resonant for all terms in the sum. In this case, however, the resonant oscillation is always $90^{\circ}$ out of phase with the steady oscillation $\exp \left[-i\left(s_{2} \omega_{\mathbf{k}_{2}} t\right)\right]$. As the coefficients $A_{\mathbf{k}_{1}, \mathbf{s}_{\mathbf{8}}, \mathbf{k}_{3}, \mathbf{k}_{\mathbf{4}}}^{\mathbf{s}_{1}, s_{2}, s_{2}}$ and $D_{\mathbf{k}_{1}, \mathbf{k}_{2}}^{s_{1}, s_{2}}$ are both imaginary, the remaining factor in the second expression is real, and we hence have the same case as discussed previously for $\operatorname{Re}\left(\overline{(}_{1} \overline{\Phi_{-k} \Phi_{k}}\right)$, the nonsteady components of the second expression yielding no contribution to the real part of the sum. The term $2 \operatorname{Re}\left(\overline{{ }_{2} \Phi_{-k 4} \Phi_{k}}\right)$ thus remains constant and has no influence on the energy transfer. 
In the same way it can be shown that the terms in the third sum indicated in (2.9), involving cubic and higher-power products of perturbations, also remain constant. Since the highest-order perturbations occurring in these products are of the fourth order, and as direct resonant excitation of the fourth-order perturbations cannot occur for the reasons considered above, the non-steady terms of the sum have the same general form

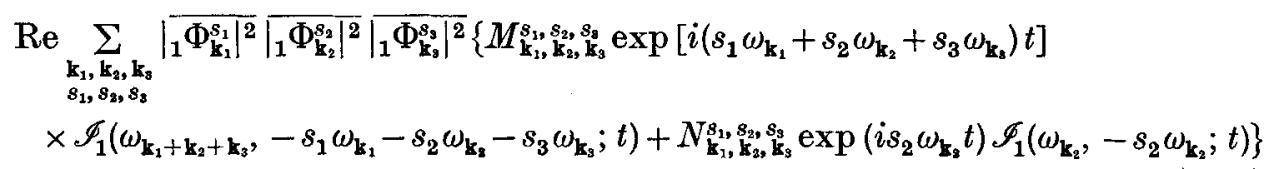

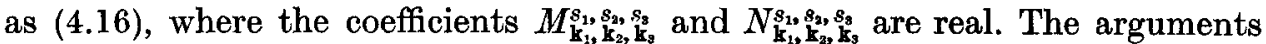
of the preceding paragraph can then be applied directly to show that (4.17) remains constant.

There remains the term $2 \operatorname{Re}\left(\overline{{ }_{1} \Phi_{-k 5} \Phi_{k}}\right)$ in the first sum in (2.9). In the differential equation for ${ }_{5} \Phi_{\mathbf{k}}$ we need consider only exciting terms containing nonsteady perturbations of the third and fourth orders, as the remaining terms can at the most give rise to simple resonant oscillations of the type $\mathscr{I}_{1}$, which yield only constant terms when correlated with the steady oscillation ${ }_{1} \Phi_{-k}$ for reasons now apparent from the discussion of the previous terms in ${ }_{6} E$. From (1.23) it follows that the perturbation equation for ${ }_{5} \Phi_{k}$ is then closely similar in structure to the equation for ${ }_{3} \Phi_{\mathrm{k}}$ and can be expressed simply in terms of the

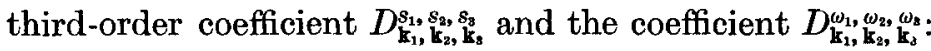

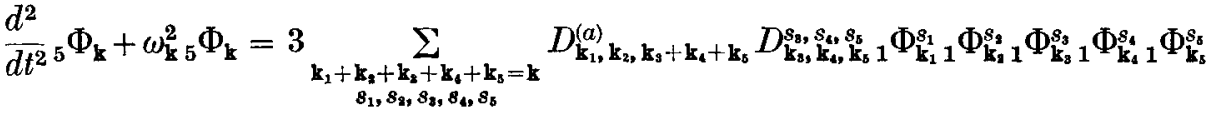

$$
\begin{aligned}
& \times \exp \left[-i\left(s_{1} \omega_{\mathbf{k}_{1}}+s_{2} \omega_{\mathbf{k}_{2}}\right) t\right] \mathscr{J}_{1}\left(\omega_{\mathbf{k}_{3}+\mathbf{k}_{4}+\mathbf{k}_{\mathbf{5}}},-s_{3} \omega_{\mathbf{k}_{3}}-s_{4} \omega_{\mathbf{k}_{4}}-s_{5} \omega_{\mathbf{k}_{6}} ; t\right) \\
& \text { + stationary terms, }
\end{aligned}
$$

where the superscript $(a) \equiv s_{1} \omega_{\mathbf{k}_{1}}, s_{2} \omega_{\mathbf{k}_{2}}, s_{3} \omega_{\mathbf{k}_{3}}+s_{4} \omega_{\mathbf{k}_{4}}+s_{5} \omega_{\mathbf{k}_{6}}$. In the mean product $\overline{ }_{1} \Phi_{-\mathbf{k} 5} \Phi_{\mathbf{k}}$ again only those terms contribute in which the six indices involved can be grouped into three conjugate pairs. Allowing for the different combinations of such pairs and the symmetry of the coefficients we find

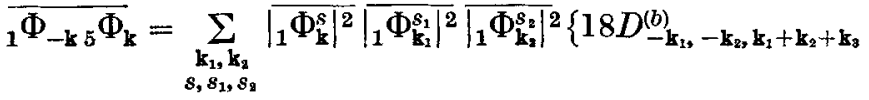

$$
\begin{aligned}
& \times D_{\mathbf{k}, \mathbf{k}_{\mathbf{k}}, \mathbf{k}_{2}}^{s, s_{1}, s_{2}} \exp \left(i s \omega_{\mathbf{k}} t\right) \mathscr{I}_{\mathbf{2}}\left(\omega_{\mathbf{k}}, s_{1} \omega_{\mathbf{k}_{1}}+s_{2} \omega_{\mathbf{k}_{2}}, \omega_{\mathbf{k}+\mathbf{k}_{1}+\mathbf{k}_{2}},-s \omega_{\mathbf{k}}-s_{1} \omega_{\mathbf{k}_{\mathbf{1}}}-s_{2} \omega_{\mathbf{k}_{2}} ; t\right) \\
& +9 D_{\mathbf{k}_{1},-\mathbf{k}_{1}, \mathbf{k}}^{(c)} D_{\mathbf{k}, \mathbf{k}_{2},-\mathbf{k}_{2}}^{s, s_{2},-s_{2}} \mathscr{I}_{2}\left(\omega_{\mathbf{k}}, 0, \omega_{\mathbf{k}},-s \omega_{\mathbf{k}} ; t\right) \exp \left(i s \omega_{\mathbf{k}} t\right) \\
& \left.+9 D_{\mathbf{k}_{1}-\mathbf{k}_{1}, \mathbf{k}_{1}}^{(d)} D_{\mathbf{k}_{\mathbf{k}_{2}}, \mathbf{k}_{2},-\mathbf{k}_{3}}^{s_{2}, \mathbf{k}_{2}} \exp \left(i s \omega_{\mathbf{k}} t\right) \mathscr{I}_{2}\left(\omega_{\mathbf{k}}, s_{1} \omega_{\mathbf{k}_{1}}-s \omega_{\mathbf{k}}, \omega_{\mathbf{k}_{1}},-s_{1} \omega_{\mathbf{k}_{1}} ; t\right)\right\} \\
& + \text { stationary terms, }
\end{aligned}
$$

where

$$
(b) \equiv-s_{1} \omega_{\mathbf{k}_{1}},-s_{\mathbf{2}} \omega_{\mathbf{k}_{2}}, s_{1} \omega_{\mathbf{k}_{\mathbf{1}}}+s_{\mathbf{2}} \omega_{\mathbf{k}_{2}}+s_{\mathbf{3}} \omega_{\mathbf{k}_{\mathbf{3}}},
$$

$$
(c) \equiv s_{1} \omega_{\mathbf{k}_{1}},-s_{1} \omega_{\mathbf{k}_{1}}, s \omega_{\mathbf{k}} \quad \text { and } \quad(d) \equiv s \omega_{\mathbf{k}},-s_{1} \omega_{\mathbf{k}_{1}}, s_{1} \omega_{\mathbf{k}_{1}}
$$

According to (3.14), the first expression in the sum increases linearly in $t$, whereas from (3.13) the increase of the last two expressions is quadratic. Again all 
non-steady components in (4.19) are entirely due to resonant interactions in which the perturbation ${ }_{5} \Phi_{\mathbf{k}}$ is excited with its natural frequencies $\pm \omega_{\mathbf{k}}$. We can thus write

$$
{ }_{5} \Phi_{\mathrm{k}}={ }_{5} \Phi_{\mathbf{k}}^{+} e^{-i \omega_{\mathbf{k}} t}+{ }_{5} \Phi_{\mathbf{k}}^{-} e^{i \omega_{\mathbf{k}} t}+\text { non-contributing terms }
$$

and obtain, after separating the components of different propagation directions in (4.19),

$$
2 \operatorname{Re}\left(\overline{{ }_{1} \Phi_{-\mathbf{k} 5} \Phi_{\mathbf{k}}}\right)=2 \operatorname{Re}\left(\overline{{ }_{1} \Phi_{-\mathrm{k} 5}^{-} \Phi_{\mathrm{k}}^{+}}\right)+2 \operatorname{Re}\left(\overline{{ }_{1} \Phi_{-\mathrm{k} 5}^{+} \Phi_{\mathrm{k}}^{-}}\right)+\text {constant terms, }
$$

where, from (3.14) and (3.13),

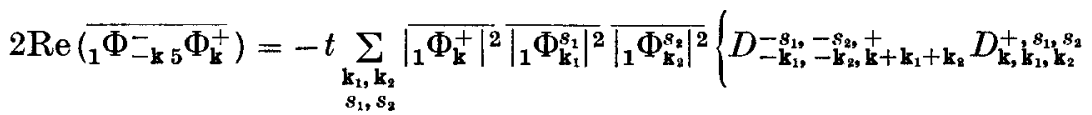

$$
\begin{aligned}
& \varkappa \frac{9 \pi}{\omega_{\mathbf{k}}\left(\omega_{\mathbf{k}}+s_{1} \omega_{\mathbf{k}_{1}}+s_{2} \omega_{\mathbf{k}_{\mathbf{2}}}\right)} \delta\left(\omega_{\mathbf{k}+\mathbf{k}_{1}+\mathbf{k}_{2}}-\omega_{\mathbf{k}}-s_{1} \omega_{\mathbf{k}_{1}}-s_{2} \omega_{\mathbf{k}_{2}}\right) \\
& \left.+D_{-\mathbf{k}_{1},-\mathbf{k}_{2}, \mathbf{k}+\mathbf{k}_{1}+\mathbf{k}_{2}}^{-s_{1},-s_{2}} D_{\mathbf{k}, \mathbf{k}_{1}, \mathbf{k}_{2}}^{+} \frac{s_{1}, s_{2}}{\omega_{\mathbf{k}}\left(\omega_{\mathbf{k}}+s_{1} \omega_{\mathbf{k}_{1}}+s_{2} \omega_{\mathbf{k}_{2}}\right)} \delta\left(\omega_{\mathbf{k}+\mathbf{k}_{1}+\mathbf{k}_{\mathbf{2}}}+\omega_{\mathbf{k}}+s_{1} \omega_{\mathbf{k}_{\mathbf{1}}}+s_{2} \omega_{\mathbf{k}_{\mathbf{2}}}\right)\right\}
\end{aligned}
$$

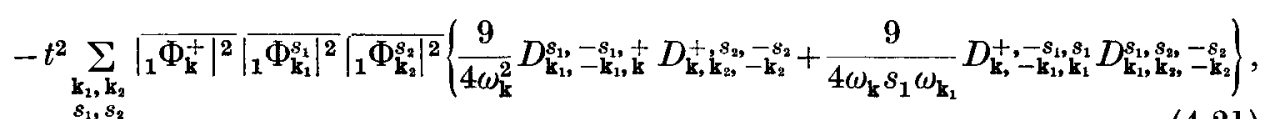

$$
\begin{aligned}
& 2 \operatorname{Re}\left(\overline{\Phi_{-\mathrm{k} 5}^{+} \Phi_{\mathrm{k}}^{-}}\right)_{i}^{\prime}=2 \operatorname{Re}\left(\overline{\Phi_{\mathrm{k}}^{-} \Phi_{-\mathrm{k}}^{+}}\right) .
\end{aligned}
$$

In (4.21) it has now been possible to replace the coefficients $D_{\mathbf{k}_{10}, \mathbf{k}_{2}, \mathbf{k}_{2}}^{\omega_{1}, \omega_{3}, \omega_{3}}$ by the coefficients $D_{\mathbf{k}_{1}, \mathbf{k}_{2}, \mathbf{k}_{2}}^{s_{1}, s_{2}, s_{2}}$, as the frequencies $\omega_{j}$ in the former coefficient satisfy the relation $\omega_{j}=s_{j} \omega_{\mathbf{k}_{j}}$ when the $\delta$-functions are non-zero. The last term in the second sum vanishes when summed over the indices $\left(\begin{array}{l}S_{1} \\ \mathbf{k}_{1}\end{array}\right)$, as terms of opposite sign in $\left(\begin{array}{l}\mathbf{s}_{1} \\ \mathbf{k}_{1}\end{array}\right)$ cancel. The first term in the second sum then cancels against the term proportional to $t^{2}$ in (4.14). Thus in the two expressions (4.14) and (4.21) representing the first sum in (2.9) finally only the sums remain which change linearly in $t$.

In order to determine the second sum in (2.9), depending on the displacement amplitudes, we note again that the non-steady terms in the first sum result entirely from resonant interactions in which the perturbations ${ }_{n} \Phi_{\mathrm{k}}$ are excited with their natural frequencies $\pm \omega_{\mathbf{k}}$; the steady higher-harmonic components with frequencies $\omega \neq \pm \omega_{\mathbf{k}}$ playing no direct part in the energy transfer. From the remark below equation (2.9), it thus follows that the non-steady components of the second sum are equal to those of the first sum.

Since the non-steady components of the perturbations are free waves of the same type as the first-order waves, we can now interpret the non-steady energy perturbations found above directly as perturbations of the two-dimensional energy spectrum.

In $\S 2$, we found for the energy of the sea

$$
E=\sum_{\mathbf{k}} \frac{\omega_{\mathbf{k}}^{2}}{g}\left|\Phi_{\mathbf{k}}\right|^{2}+\Delta E
$$

where

$$
\Phi_{\mathbf{k}}(t)={ }_{1} \Phi_{\mathbf{k}}+{ }_{2} \Phi_{\mathbf{k}}+{ }_{3} \Phi_{\mathbf{k}}+\ldots
$$


and $\Delta E$ contains only cubic and higher-power products which were found to remain constant up to the sixth perturbation order. In integral form, (4.22) becomes

where

$$
E=\iint_{-\infty}^{+\infty} \hat{F}(\mathbf{k}) d k_{x} d k_{y}+\Delta E
$$

is the general (non-linear) instantaneous space spectrum of the sea. In the linear approximation, the Fourier components ${ }_{1} \Phi_{\mathbf{k}}(t) \exp (i \mathbf{k} . \tilde{\mathbf{x}})$ are composed solely of two progressive sinusoidal waves

$$
{ }_{\mathbf{1}} \Phi_{\mathbf{k}}^{+} \tilde{\mathbf{x}} \exp \left[i(\mathbf{k})-i \omega_{\mathbf{l}} t\right] \text { and }{ }_{1} \Phi_{\mathbf{k}}^{-} \exp \left[i(\mathbf{k} \tilde{\mathbf{x}})+i \omega_{\mathbf{k}} t\right]
$$

so that we could separate the spectrum ${ }_{2} \hat{F}(\mathbf{k})$ into two components ${ }_{2} F(\mathbf{k})$ and ${ }_{2} F(-\mathbf{k})$, with ${ }_{2} \hat{F}(\mathbf{k})=\frac{1}{2}\left\{{ }_{2} F(\mathbf{k})+{ }_{2} F(-\mathbf{k})\right\}$, corresponding to the two directions of propagation of the waves. In contrast to ${ }_{2} \hat{F}(\mathbf{k})$, the spectrum ${ }_{2} F(\mathbf{k})$ describes the sea to the first order completely. The next spectral perturbation term ${ }_{4} \hat{F}(\mathbf{k})$ remains constant and need not be considered. The term ${ }_{6} \hat{F}(\mathbf{k})$ contains nonstationary components which again depend only on progressive waves of the type ${ }_{n} \Phi_{\mathbf{k}}^{ \pm} \exp \left[i(\mathbf{k} \tilde{\mathbf{x}}) \mp i \omega_{\mathbf{k}} t\right]$, so that we can write

$$
{ }_{6} \hat{F}(\mathbf{k})=\frac{1}{2}\left\{{ }_{6} F(\mathbf{k})+{ }_{6} F(-\mathbf{k})\right\}+\text { stationary terms. }
$$

The relation between ${ }_{6} F(\mathbf{k})$ and the terms entering in ${ }_{6} E$ is then found from (2.9), (4.13), (4.20), (4.23) and (4.25),

$$
\iint_{6} F(\mathbf{k}) d k_{x} d k_{y}=\sum_{\mathbf{k}} \frac{2 \rho \omega_{\mathbf{k}}^{2}}{g}\left\{\overline{\left.{ }_{3} \Phi_{\mathbf{k}}^{+}\right|^{2}}+2 \operatorname{Re}\left(\overline{{ }_{1} \Phi_{-\mathbf{k} 5}^{-} \Phi_{\mathbf{k}}^{+}}\right)\right\}
$$

From (4.14) and (4.21) we then obtain

$$
\begin{aligned}
& { }_{6} F(\mathbf{k})=t \iiint \int_{-\infty}^{+\infty} \sum_{s^{\prime}, s^{\prime \prime}, s^{\prime \prime}} \frac{3 \pi g^{2}}{4 \rho^{2} \omega_{\mathbf{k}^{\prime}}^{2} \omega_{\mathbf{k}^{\prime \prime}}^{2} \omega_{\mathbf{k}-\mathbf{k}^{\prime}-\mathbf{k}^{\prime \prime}}^{2}}\left(D_{\mathbf{k}^{\prime}, \mathbf{k}^{\prime \prime}, s^{\prime \prime}-\mathbf{k}^{\prime}-\mathbf{k}^{\prime}}^{s^{\prime}}\right)^{2} \\
& \times \delta\left(\omega_{\mathbf{k}}+s^{\prime} \omega_{\mathbf{k}^{\prime}}+s^{\prime \prime} \omega_{\mathbf{k}^{\prime}}+s^{\prime \prime \prime} \omega_{\mathbf{k}-\mathbf{k}^{\prime}-\mathbf{k}^{*}}\right)_{2} F\left(s^{\prime} \mathbf{k}^{\prime}\right)_{2} F\left(s^{\prime \prime} \mathbf{k}^{\prime \prime}\right) \\
& \times_{3} F\left(s^{\prime \prime \prime}\left[\mathbf{k}-\mathbf{k}^{\prime}-\mathbf{k}^{\prime \prime}\right]\right) d k_{x}^{\prime} d k_{y}^{\prime} d k_{x}^{\prime \prime} d k_{y}^{\prime \prime} \\
& -t \iiint \int_{-\infty}^{+\infty} \sum_{s^{\prime}, s^{\prime \prime}, s^{\prime \prime}} \frac{9 \pi g^{2}}{4 \rho^{2} \omega_{\mathbf{k}^{\prime}}^{2} \omega_{\mathbf{k}^{*}}^{2}\left(\omega_{\mathbf{k}}+s^{\prime} \omega_{\mathbf{k}^{\prime}}+s^{\prime \prime} \omega_{\mathbf{k}^{\prime \prime}}\right) \omega_{\mathbf{k}}} D_{-\mathbf{k}^{\prime} ;-\mathbf{k}^{\prime \prime}, s^{\prime \prime}+\mathbf{k}^{\prime}+\mathbf{k}^{*}}^{-s^{\prime \prime}} \\
& \times D_{\mathbf{k}, \mathbf{k}^{\prime}, \mathbf{k}^{\prime \prime}}^{+} \delta\left(\omega_{\mathbf{k}}+s^{\prime} \omega_{\mathbf{k}^{\prime}}+s^{\prime \prime} \omega_{\mathbf{k}^{\prime \prime}}-s^{\prime \prime \prime} \omega_{\mathbf{k}+\mathbf{k}^{\prime}+\mathbf{k}^{\prime \prime}}\right) \\
& \times_{2} F(\mathbf{k})_{2} F\left(s^{\prime} \mathbf{k}^{\prime}\right)_{2} F\left(s^{\prime \prime} \mathbf{k}^{\prime \prime}\right) d k_{x}^{\prime} d k_{y}^{\prime} d k_{x}^{\prime \prime} d k_{y}^{\prime \prime} \text {. }
\end{aligned}
$$

On account of the $\delta$-functions in the integrands, the quadruple integrals are in reality only triple integrals over hypersurfaces in the four-dimensional $\left(\mathbf{k}^{\prime} \times \mathbf{k}^{\prime \prime}\right)$ space.

In order to carry out the sums over the sign indices we note that successive application of (4.6) yields the inequality

$$
\omega_{\mathbf{k}_{1}}-\omega_{\mathbf{k}_{\mathbf{z}}}-\omega_{\mathbf{k}_{3}} \leqslant \omega_{\mathbf{k}_{1}+\mathbf{k}_{2}+\mathbf{k}_{8}} \leqslant \omega_{\mathbf{k}_{\mathbf{1}}}+\omega_{\mathbf{k}_{\mathbf{2}}}+\omega_{\mathbf{k}_{8}}
$$

the equality signs holding only for trivial cases in which the corresponding coefficients in (4.27) vanish. On account of (4.28), we need consider only those sign combinations in (4.27) in which two of the frequencies in the $\delta$-functions 
are positive and two negative. This gives three combinations for each integral, the combinations for the first integral yielding the same result for reasons of symmetry. Hence finally $\dagger$

$$
\begin{array}{r}
\frac{\partial F(\mathbf{k})}{\partial t}=\iiint \int_{-\infty}^{+\infty} F\left(\mathbf{k}^{\prime}\right) F\left(\mathbf{k}^{\prime \prime}\right) F\left(\mathbf{k}^{\prime}+\mathbf{k}^{\prime \prime}-\mathbf{k}\right) T_{1}\left(\mathbf{k}^{\prime}, \mathbf{k}^{\prime \prime}, \mathbf{k}^{\prime}+\mathbf{k}^{\prime \prime}-\mathbf{k}\right) d k_{x}^{\prime} d k_{y}^{\prime} d k_{x}^{\prime \prime} d k_{y}^{\prime \prime} \\
-F(\mathbf{k}) \iiint \int_{-\infty}^{+\infty} F\left(\mathbf{k}^{\prime}\right) F\left(\mathbf{k}^{\prime \prime}\right) T_{2}\left(\mathbf{k}, \mathbf{k}^{\prime}, \mathbf{k}^{\prime \prime}\right) d k_{x}^{\prime} d k_{y}^{\prime} d k_{x}^{\prime \prime} d k_{y}^{\prime \prime},
\end{array}
$$

where

and

$$
T_{1}\left(\mathbf{k}_{1}, \mathbf{k}_{2}, \mathbf{k}_{3}\right)=\frac{9 \pi g^{2}}{4 \rho^{2} \omega_{\mathbf{k}_{1}}^{2} \omega_{\mathbf{l}_{3}}^{2} \omega_{\mathbf{k}_{3}}^{2}}\left(D_{\mathbf{k}_{\mathbf{k}_{4}}, \mathbf{k}_{\mathbf{2}_{2}}-\mathbf{k}_{3}}^{+}\right)^{2} \delta\left(\omega_{\mathbf{k}_{1}+\mathbf{k}_{2}-\mathbf{k}_{3}}-\omega_{\mathbf{k}_{1}}-\omega_{\mathbf{k}_{2}}+\omega_{\mathbf{k}_{3}}\right)
$$

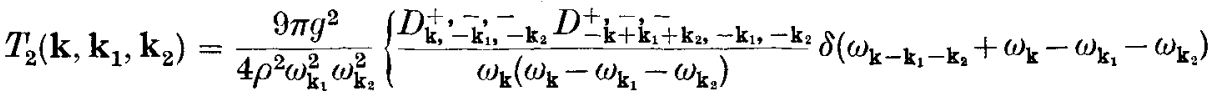

$$
\begin{aligned}
& +\frac{D_{\mathbf{k}, \mathbf{i}_{\mathbf{k}^{\prime}},-\mathbf{k}_{\mathbf{2}}}^{+} D_{-\mathbf{k}-\mathbf{k}_{1}+\mathbf{k}_{2}, \mathbf{k}_{1},-\mathbf{k}_{2}}^{+}}{\omega_{\mathbf{k}}\left(\omega_{\mathbf{k}}+\omega_{\mathbf{k}_{1}}-\omega_{\mathbf{k}_{2}}\right)} \delta\left(\omega_{\mathbf{k}+\mathbf{k}_{1}-\mathbf{k}_{2}}-\omega_{\mathbf{k}}-\omega_{\mathbf{k}_{1}}+\omega_{\mathbf{k}_{2}}\right)
\end{aligned}
$$

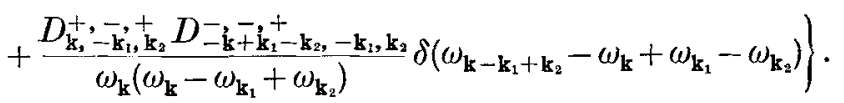

In (4.29), the time derivative has been taken and the perturbation indices dropped. Whereas the validity of the original equation (4.27) is restricted to $t$ sufficiently small to ensure ${ }_{6} F(\mathbf{k}) \ll{ }_{2} F(\mathbf{k}),(4.29)$ is valid for all $t$ provided the basic condition of a first-order Gaussian sea is not violated. Although there is no doubt that the non-linear interactions not only transfer energy but will also gradually destroy the initial Gaussian property of the sea, it follows from our derivation that the influence of the latter process on the energy transfer is negligible. Since the non-stationary components of ${ }_{6} E$ were determined alone by covariance products between perturbation amplitudes having the same wavenumber, the influence of non-Gaussian couplings between perturbation amplitudes with different wave-numbers is necessarily a higher-order effect which can be neglected for the time periods normally involved in the energy transfer process.

Equation (4.29) can be checked by the law of energy conservation, which requires that

$$
\iint_{-\infty}^{+\infty} \frac{\partial}{\partial t} F(\mathbf{k}) d k_{x} d k_{y}=0
$$

For arbitrary $F(\mathbf{k})$ this gives the condition

$$
\sum_{\text {Perm. in } \mathbf{k}_{j}}\left[T_{1}\left(\mathbf{k}_{1}, \mathbf{k}_{2}, \mathbf{k}_{3}\right)-T_{2}\left(\mathbf{k}_{1}, \mathbf{k}_{2}, \mathbf{k}_{3}\right)\right]=0,
$$

which is equivalent to the relation

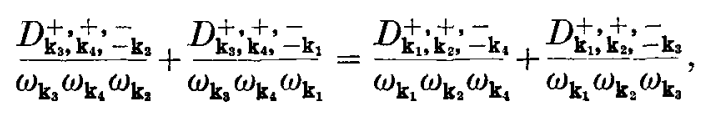

$\dagger$ Equation (4.29) was first cited and discussed briefly in connexion with the complete equation for the energy balance of a spectrum in the author's 1960 paper. A short outline of the derivation is given in the 1961 paper. 
for arbitrary $\mathbf{k}_{j}$ satisfying the interaction conditions

$$
\begin{gathered}
\mathbf{k}_{1}+\mathbf{k}_{2}=\mathbf{k}_{3}+\mathbf{k}_{4}, \\
\omega_{\mathbf{k}_{1}}+\omega_{\mathbf{k}_{2}}=\omega_{\mathbf{k}_{3}}+\omega_{\mathbf{k}_{4}} .
\end{gathered}
$$

Equation (4.33) was checked numerically for the case $h=\infty$.

By suitable transformation of the integration variables in the second integral, we can express both integrals in (4.29) in terms of a common $\delta$-function. Applying further (4.33), we obtain

$$
\begin{aligned}
& \frac{\partial F(\mathbf{k})}{\partial t}=\iiint \int_{-\infty}^{+\infty} \frac{9 \pi g^{2} D_{\mathbf{k}^{\prime}, \mathbf{k}^{\prime \prime}, \mathbf{k}-\mathbf{k}^{\prime}-\mathbf{k}^{\prime \prime}}^{+}}{4 \rho^{2} \omega_{\mathbf{k}^{\prime}} \omega_{\mathbf{k}^{\prime \prime}} \omega_{\mathbf{k}^{\prime}+\mathbf{k}^{\prime \prime}-\mathbf{k}}^{+}}\left\{\frac{D_{\mathbf{k}^{\prime}, \mathbf{k}^{\prime \prime}}^{+},-\mathbf{k}}{\omega_{\mathbf{k}^{\prime}} \omega_{\mathbf{k}^{*}} \omega_{\mathbf{k}}} F\left(\mathbf{k}^{\prime}\right) F\left(\mathbf{k}^{\prime \prime}\right)\left[F(\mathbf{k})-F\left(\mathbf{k}^{\prime}+\mathbf{k}^{\prime \prime}-\mathbf{k}\right)\right]\right. \\
& -\frac{D_{\mathbf{k}^{\prime}+\mathbf{k}^{\prime \prime}=\mathbf{k}, \mathbf{k},-\mathbf{k}^{\prime \prime}}^{+}}{\omega_{\mathbf{k}^{\prime}+\mathbf{k}^{\prime \prime}-\mathbf{k}} \omega_{\mathbf{k}}\left(\omega_{\mathbf{k}^{\prime \prime}}\right.} F\left(\mathbf{k}^{\prime \prime}\right) F\left(\mathbf{k}^{\prime}+\mathbf{k}^{\prime \prime}-\mathbf{k}\right)\left[F(\mathbf{k})-F\left(\mathbf{k}^{\prime}\right)\right] \\
& \left.-\frac{D_{\mathbf{k}^{\prime}+\mathbf{k}^{\prime \prime}-\mathbf{k}, \mathbf{k},-\mathbf{k}^{\prime}}^{+}}{\omega_{\mathbf{k}^{\prime}+\mathbf{k}^{\prime \prime}-\mathbf{k}} \omega_{\mathbf{k}} \omega_{\mathbf{k}^{\prime}}} F\left(\mathbf{k}^{\prime}\right) F^{\prime}\left(\mathbf{k}^{\prime}+\mathbf{k}^{\prime \prime}-\mathbf{k}\right)\left[F(\mathbf{k})-F\left(\mathbf{k}^{\prime \prime}\right)\right]\right\} \\
& \times \delta\left(\omega_{\mathbf{k}^{\prime}}+\omega_{\mathbf{k}^{\prime \prime}}-\omega_{\mathbf{k}^{\prime}+\mathbf{k}^{\prime \prime}-\mathbf{k}}-\omega_{\mathbf{k}}\right) d k_{x}^{\prime} d k_{y}^{\prime} d k_{x}^{\prime \prime} d k_{y}^{\prime \prime} .
\end{aligned}
$$

Equation (4.36) is more suitable than (4.29) for computational purposes and also for discussing certain aspects of the energy transfer. The last two terms in (4.36) and (4.31) differ only in that the integration variables are interchanged. They are written separately, however, in order to facilitate the following discussion.

\section{Interpretation and discussion}

Equations (4.29) and (4.36) can be interpreted in terms of quadruple interactions between three 'active' wave components, which determine the interaction rate, and a 'passive' fourth component, which receives energy from the first three components but has no direct influence on the interaction. In (4.29), the first integral then expresses the energy gained by the component $\uparrow \mathbf{k}$ from all quadruple interactions in which $\mathbf{k}$ represents a ' passive' component, whereas the second integral expresses the energy lost by all interactions in which $\mathbf{k}$ is one of the three 'active' components. According to (4.30), we have $T_{1} \geqslant 0$, so that the energy gain is always positive (or zero). Although from (4.32) the total energy loss integrated over $\mathbf{k}$ is also positive, this is not necessarily the case for a particular value of $\mathbf{k}$, so that the second integral may for some $\mathbf{k}$ represent an energy gain rather than a loss.

The expression for the energy gain in (4.29) is independent of, but the loss proportional to, the value of the spectrum at $\mathbf{k}$. The transfer process will thus generally tend to reduce sharp peaks in the spectrum and redistribute the energy more uniformly over all wave-numbers. Thus for wind-generated seas we may expect an energy flux from high to low wave-numbers in the initial period of development, when most of the energy is concentrated in the high wave-number region, and after development of the peak at low wave-numbers an energy flux in the reverse direction from low to high wave-numbers.

The alternative expression for the energy balance in the form (4.36) is obtained by regrouping the transfer components of the interactions. An interaction

$\dagger$ In accordance with the definition of $F(\mathbf{k})$, the component $\mathbf{k}$ refers always to the wavecomponent travelling in the positive $\mathbf{k}$-direction. 
between a group of four wave-numbers can take place if, and only if, the wave-numbers can be separated into two pairs, say $\left(\mathbf{k}_{1}, \mathbf{k}_{2}\right)$ and $\left(\mathbf{k}_{3}, \mathbf{k}_{4}\right)$, which satisfy the interaction conditions (4.34) and (4.35). If both conditions are satisfied, all four quadruple interactions corresponding to the four possibilities of choosing one 'active' and three 'passive' components take place. If the net energy change resulting from all four interactions is then evaluated for a particular wave-number $\mathbf{k}$ in the group, the total rate of change of the spectrum at $k$ can be expressed in the form (4.36) by integrating this net change over all groups of interacting wave-numbers. From (4.36), a number of interesting properties of the transfer process can immediately be inferred. (1) If all four values of the spectrum for an interacting group are equal, the net energy transfer of the group is balanced. Hence, in particular, the total energy transfer vanishes for the limiting case of a white, isotropic spectrum. (2) If the spectral values at three points of an interacting group are equal, we find, using (4.33), that the value of the spectrum at the fourth point changes in the direction of the first three values. A more general statement of this tendency to equidistribution can be made for arbitrary values of the spectrum if the three coefficient products in the integral (4.36) are negative not only in the sum (on account of (4.33)), but also individually. In this case the net effect of all four interactions of a group is always to reduce the maximum and increase the minimum of the spectrum at the four points considered. (3) The net energy transfer of a group is balanced if the wave-number pairs $\left(\mathbf{k}_{1}, \mathbf{k}_{2}\right),\left(\mathbf{k}_{3}, \mathbf{k}_{4}\right)$ are equal, irrespective of the values of the spectrum. $\dagger$ In particular, this is always the case for a unidirectional spectrum. Equation (4.34) then becomes the scalar condition

$$
k_{1}+k_{2}=k_{3}+k_{4}
$$

which can be satisfied only if one wave-number pair lies within the other, say

$$
k_{1} \leqslant k_{3} \leqslant k_{4} \leqslant k_{2}
$$

It can then readily be shown that, since $\omega_{\mathbf{k}}=\Omega(k)$ is convex,

$$
\Omega\left(k_{1}\right)+\Omega\left(k_{2}\right) \leqslant \Omega\left(k_{3}\right)+\Omega\left(k_{4}\right),
$$

the equality sign holding only for $\left(k_{1}, k_{2}\right)=\left(k_{3}, k_{4}\right)$. For a unidirectional spectrum the interaction conditions (4.34) and (4.35) thus have only trivial solutions and the non-linear energy transfer vanishes (or, more precisely, is of smaller order than (4.29)).

An indication of the order of magnitude of the energy flux for the general case of a two-dimensional spectrum can be obtained by dimensional analysis of (4.29). If a characteristic wave height $\zeta_{0}$, wavelength $\lambda_{0}$ and wave period $T_{0}$ (dependent on $\lambda_{0}$ through $T_{0}^{2} g \sim \lambda_{0}$ ) are introduced, we find for the characteristic time $T$ of the energy transfer

$$
T \sim T_{0} \vartheta^{-4}
$$

where $\vartheta$ is the root mean square wave slope. Assuming the proportionality factor equal to unity (obviously a very crude procedure) we find for $T_{0}=10 \mathrm{sec}$ and

$\dagger$ It has been shown by Longuet-Higgins \& Phillips (1962) that in this case the tertiary waves lead to a change in phase velocity of the primary wave. 
$\vartheta=0 \cdot 1$, say, $T=28 \mathrm{~h}$, which is comparable in magnitude to the development periods of wave spectra.

A more reliable estimate of the magnitude and form of the non-linear energy flux has been obtained by computing (4.36) for a typical fully developed spectrum (Hasselmann 1961). Although the computer used, an IBM 650, was too small to achieve great accuracy, an energy flux was found, as expected, from the spectral peak at low wave-numbers to the low-energy region of dissipation at higher wave-numbers. The characteristic time scales of the energy flux were found to be of the order of a few fractions of an hour for the shorter waves and a few hours for the longer waves.

It is hoped to carry out a more detailed investigation of the non-linear energy transfer for a wider variety of cases, using larger computer facilities. Apart from influencing the final equilibrium status of fully developed wave spectra, it is possible that the non-linear interactions may also play an important role in the damping of swell travelling through local wind seas (predominance of the energy-loss integral in (4.29)), the scattering, including back-scattering, of waves leaving storm areas, and the aforementioned generation of very long waves by interactions between the shorter wind-generated waves.

The author is indebted to Professor M. S. Longuet-Higgins for valuable comments on the first draft of this paper.

\section{REFERENCES}

HasselmanN, K. 1960 Sehiffstechnik, 7, 191-5.

Hasselmann, K. 1961 Proc. Conf. Ocean Wave Spectra, Easton, Md. (to be published).

LoNGUET-Higgins, M. S. 1962 J. Fluid Mech. 12, 321.

Longuet-Higgrns, M. S. \& Phinlips, O. M. 1962 J. Fluid Mech. 12, 333.

Munk, W. H. 1961 Proc. Conf. Ocean Wave Spectra, Easton, Md. (to be published).

Phillips, O. M. 1957 J. Fluid Mech. 2, 417-45.

Phiclits, O. M. 1960 J. Fluid Mech. 9, 193-217.

Trck, L. J. 1958 N.Y. Univ. Coll. Eng. Sci. Paper No. 11. 